\title{
A critical review of the current technologies in wastewater treatment plants by using hydrodynamic cavitation process: principles and applications
}

\author{
Giuseppe Mancuso $^{1}$ (D) $\cdot$ Michela Langone ${ }^{2} \cdot$ Gianni Andreottola $^{2}$ \\ Received: 20 February 2019 / Accepted: 14 January 2020 / Published online: 27 January 2020 \\ (C) Springer Nature Switzerland AG 2020
}

\begin{abstract}
In the last decade, hydrodynamic cavitation (HC) was increasingly used in the field of wastewater treatment. Due to its oxidative capability, $\mathrm{HC}$ was applied to treat aqueous effluents polluted by organic, toxic and bio-refractory contaminants, whereas its mechanical and chemical effects have allowed to disintegrate cells of microorganisms in biological applications. Due to their geometries, $\mathrm{HC}$ can be detected in some reactors, in which a variation of hydraulic parameters in the fluid such as flow pressure and flow velocity is induced. HC process involves the formation, growth, implosion and subsequent collapse of cavities, occurring in a very short period of time and releasing large magnitudes of power. In this paper, the vast literature on $\mathrm{HC}$ is critically reviewed, focusing on the basic principles behind it, in terms of process definition and analysis of governing mechanisms of both HC generation and pollutants degradation. The influence of various parameters on HC effectiveness was assessed, considering fluid properties, construction features of $\mathrm{HC}$ devices and technological aspects of processes. The synergetic effect of $\mathrm{HC}$ combined with chemicals or other techniques was discussed. An overview of the main devices used for HC generation and different existing methods to evaluate the cavitation effectiveness was provided. Knowledge buildup and optimization for such complex systems from mathematical modeling was highlighted.
\end{abstract}

Keywords Hydrodynamic cavitation · Computational fluid dynamics · Modeling · Pollutant degradation · Wastewater treatment

Highlights

- Factors influencing the hydrodynamic cavitation (HC) efficiency.

- Classification of HC mechanisms on degradation of pollutants.

- Methods to evaluate HC efficiency for degradation of pollutants.

- Mathematical models to simulate fluid dynamics into HC devices.

Giuseppe Mancuso

g.mancuso@unibo.it

Michela Langone

michela.langone@unitn.it

Gianni Andreottola

gianni.andreottola@unitn.it

1 Department of Agricultural and Food Sciences, Alma Mater Studiorum, University of Bologna, viale Giuseppe Fanin 50, 40127 Bologna, Italy

2 Department of Civil, Environmental and Mechanical Engineering, University of Trento, via Mesiano 77, 38123 Trento, Italy

\section{Introduction}

In the last years, many researchers worldwide published scientific papers on $\mathrm{HC}$ process. Initially, the main research topic was the comprehension of $\mathrm{HC}$ negative effects on hydraulic machineries, such as turbines, pumps, valves, etc. [1-6]. With this purpose, researchers have dedicated many efforts to establish the basic mechanisms in $\mathrm{HC}$ process generation, in order to overcome some very serious problems related to hydraulic machineries, such as vibrations, erosions and noises $[1,5]$. Therefore, many studies have been carried out in order to identify methodologies and parameters aimed to predict and, then, avoid cavitation inception [7].

Recently, some worldwide widespread problems such as water resources availability, population increase in developing countries and the related water consumption, or more strict legislations on water quality, led to the use of $\mathrm{HC}$ as an innovative technique in the field of wastewater treatment. Figure 1 shows the significant increase in the number of peer-reviewed publications indexed by Scopus databases on innovative wastewater treatments and, moreover, on the application of 


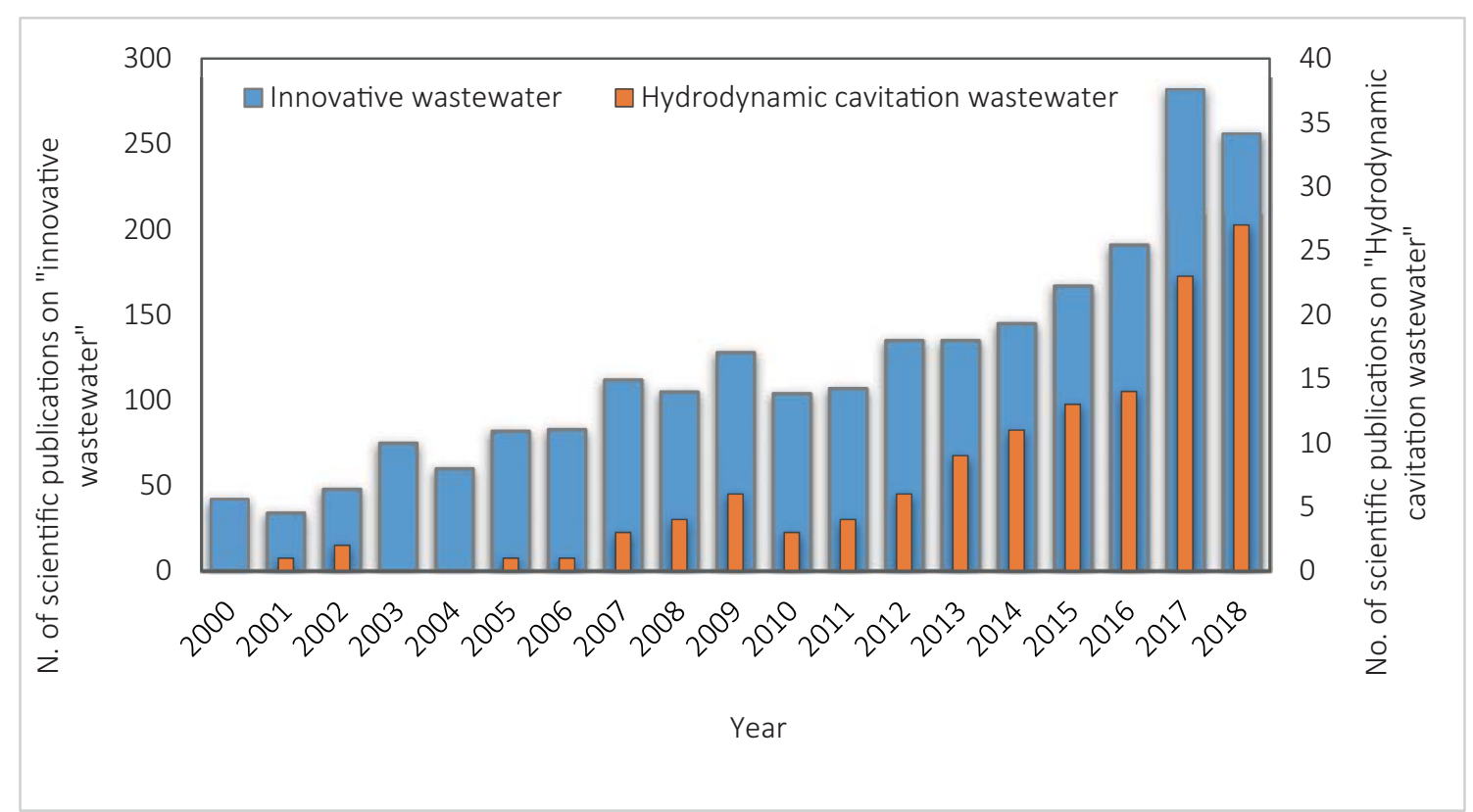

Fig. 1 Increase in the number of peer-reviewed publications listed in the databases of Scopus from 2000 to 2018 with "innovative wastewater" and "hydrodynamic cavitation wastewater" as key words

$\mathrm{HC}$ as an innovative technique in the field of wastewater treatment.

It has been widely proven that $\mathrm{HC}$ is characterized by a very high oxidative capacity, which favours the use of $\mathrm{HC}$, used alone or in combination with other techniques, in a large number of applications in the field of wastewater treatment, ranging from biological processes such as cellular disruption to chemical treatments such as the oxidation of organic pollutants in aqueous effluents, including bio-refractory and toxic chemicals [8-10].

In the field of wastewater, the inability of the most advanced oxidation processes (AOP) to completely degrade complex compounds has generally led to the application of cavitation to overcome this limit. For this purpose, the effectiveness of the cavitation process in wastewater treatment has been widely demonstrated using acoustic cavitation (AC) [11, 12]. However, in the last decade, restrictions due to high costs related to AC applications have been overcome by the development of HC technologies [13]. Moreover, when compared with other conventional treatment techniques, $\mathrm{HC}$ has proved to be characterized by greater pollutants removal efficiencies, lower energy requirements $[14,15]$ and fewer secondary contamination problems.

$\mathrm{AC}$ has been widely applied to treat wastewater. On the contrary, HC has been applied in a lower number of scientific papers, but it showed promising results in various fields in WWTPs, such as biological treatments (activated sludge [16-19], pre-treatment of biomasses before anaerobic digestion [20], denitrification processes [9], reduction of activated sludge [15], enhancement of sludge dewaterability [21]), removal of toxic carcinogens and dyes [8], pharmaceutical products, toxic cyanobacteria, bacteria and viruses [22].

\section{Brief background of cavitation process}

Cavitation can be defined as the phenomena of generation (inception), growth, coalescence, fission, travelling and implosive collapse of cavitation bubbles or cavities (Fig. 2) within a liquid in less than a few microseconds. The result is a very high local temperature and pressure [23, 24], which induce physical and chemical effects, in addition to the mechanical ones.

According to its generation pathway, cavitation can be classified into acoustic cavitation (AC), hydrodynamic cavitation (HC), optical cavitation (OC), particle cavitation (PC) and steam bubbles cavitation. $\mathrm{OC}$ and $\mathrm{PC}$ occur when a liquid is irradiated with high intensity light or by means of a laser. In OC, photons are used to break the liquid, while in PC other types of elementary particles, such as protons and neutrinos, can accomplish a breakdown in the liquid [25]. In AC and HC, cavities are generated as a result of local pressure drops, due to pressure and speed fluctuations, respectively. Steam cavitation occurs when direct injection of steam into sub-cooled water is provided, producing collapse conditions similar to $\mathrm{HC}$ and $\mathrm{AC}$ [26]. Although all types of cavitation generate cavitation bubbles, it was found that mainly $\mathrm{AC}$ and $\mathrm{HC}$ are able to bring to the desired chemical changes in the treated matrixes in the environmental [27, 28]. 

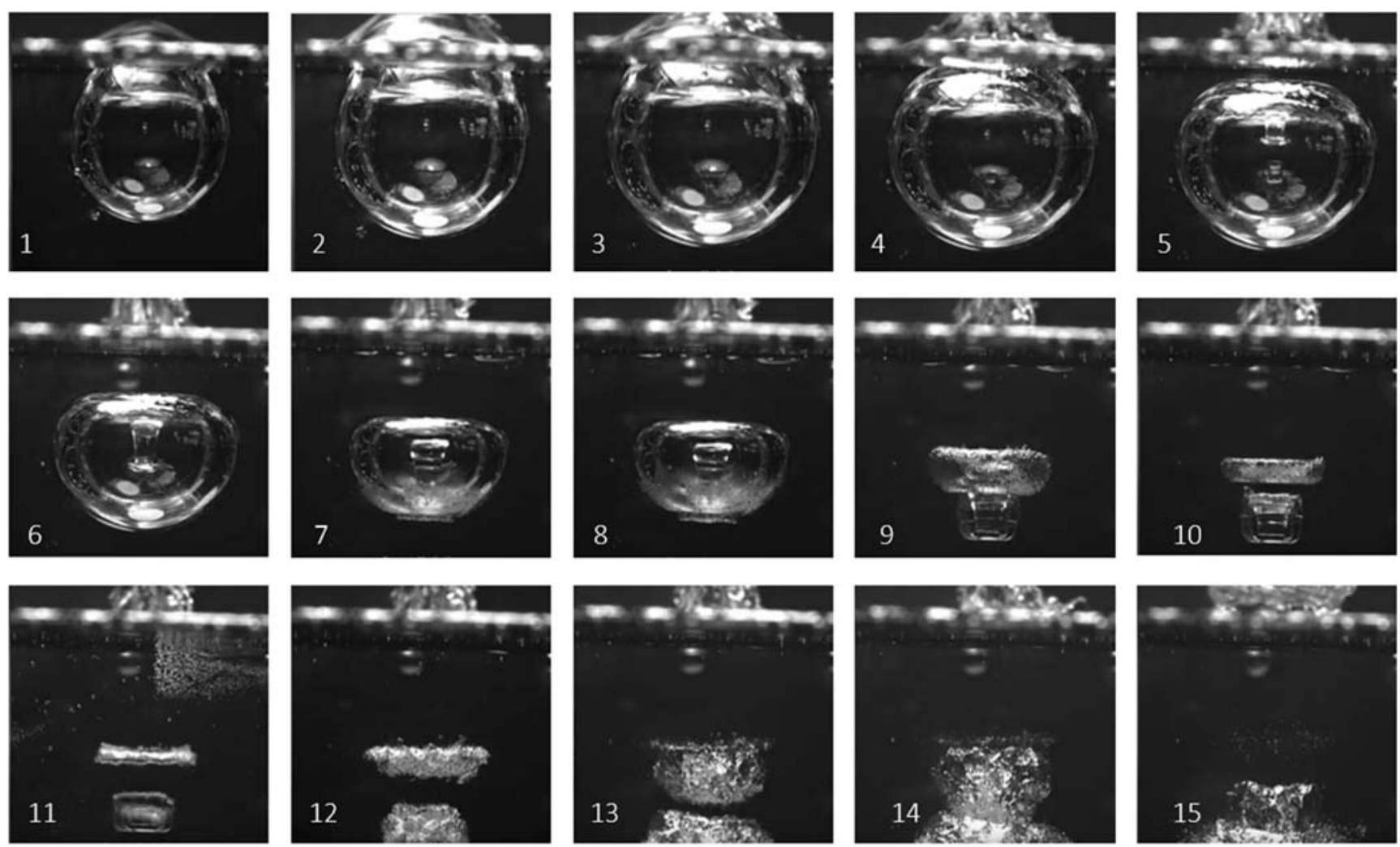

Fig. 2 Graphic illustration of growth, implosion and collapse of bubbles in the cavitation process. $1-3$ : static pressure $<$ vapor pressure $\Rightarrow$ vapor bubble grows at low pressure; 4: static pressure $=$ vapor pressure $\Rightarrow$ no

further growth of bubbles; 5-15: static pressure > vapor pressure $\Rightarrow$ implosion and collapse; 9-15: micro-jets

Afterwards, the well-known thermodynamic concept of vapor pressure will be used to explain the physical meaning of the cavitation process, based on local pressure drops. As shown in Fig. 3, in the water phase diagram the curve from the triple point to the critical point separates the liquid and vapor domains. Crossing that curve is representative of a reversible transformation in static or equilibrium conditions.

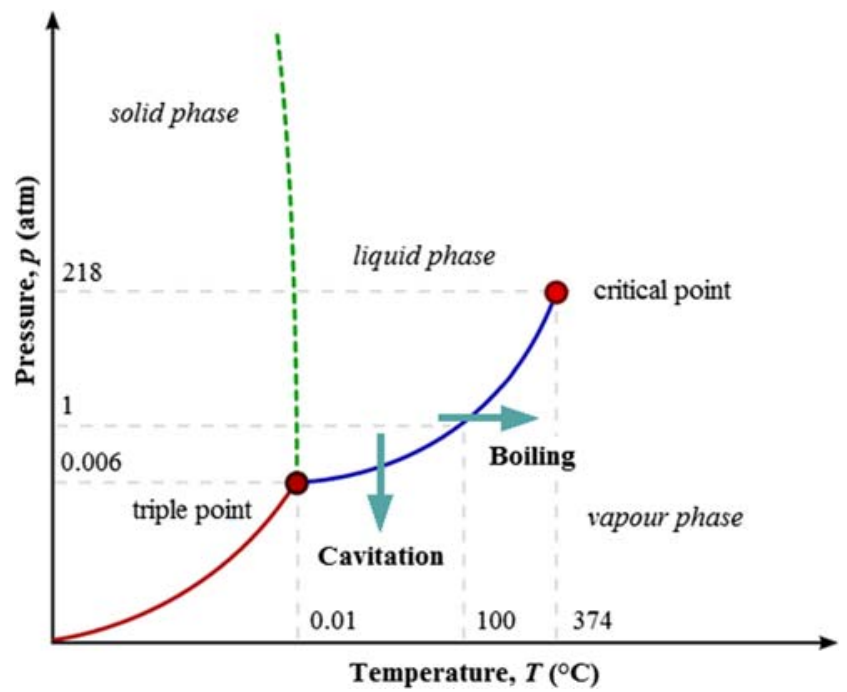

Fig. 3 - Phase diagram of water (adapted from Brennen [29])
The phase transformations from the liquid phase to the vapor phase (evaporation) or vice versa from the vapor phase to the liquid phase (condensation) take place at a specific pressure value, known as vapor pressure, $p v$, for each temperature value. Cavitation is very similar to boiling, except that the main driving mechanism is not a change in temperature, but a change in pressure. After cavitation, bubbles grow into the liquid in low-pressure regions and can be transported by convection to higher-pressure regions, where they can collapse [30].

When cavitation occurs, it is possible to observe a multiphase condition into the fluid with gas (vapor and air) and liquid. If the pressure of the mixture is equal to saturation pressure, vapour cavitation occurs. On the contrary, for pressures lower than atmospheric one, a release of air from the liquid can be observed. Since vacuum creation and vacuum filling with vapour and/or air take place simultaneously, the instantaneous saturation of the vacuum can presumably be justified [30].

It is widely accepted that cavitation inception is strongly influenced by water quality, especially in relation to the concentration of nuclei into water. Occurrence of cavitation bubbles starts from weak points into the water, namely bubbles nuclei, which break the bond between the water molecules. These nuclei are generally small gas bubbles or solid nuclei. 
Despite some differences in the generation of cavitation bubbles using $\mathrm{AC}$ and $\mathrm{HC}$, the principles governing the hydrodynamic and acoustic growth of bubbles are the same. In $\mathrm{AC}$, when the ultrasonic wave propagates in the fluid, it generates compressions and rarefactions. The compression cycles exert a positive pressure on the liquid, pushing the molecules together, while the rarefaction cycle exerts a negative pressure by pulling the molecules from one another. Due to low pressure, cavitation bubbles are generated in the rarefaction regions. These bubbles grow in successive cycles, reaching an unstable diameter and collapsing violently [31]. In HC, due to the presence of a restriction into the fluid, that usually involves a reduction in the cross-section of the flow, low pressures are reached, with consequent generation of cavitation bubbles. Downstream of the constriction, as the cross section expands, the pressure of the flow is restored and this leads to the growth of the bubbles, reaching an unstable diameter, whereby the cavities collapse violently [32]. The bubbles that occur in a cavitating flow are not perfectly spherical. However, it is often said that spherical analysis shows a satisfactory dynamic modeling of higher level bubbles. Furthermore, it is often assumed that there is a growth of isothermal bubbles up to their maximum radius, in which the bubbles are mainly filled with vapor and/or air at equilibrium at room temperature [33].

Many studies have been conducted to investigate the dynamics of bubble collapse [34], showing the presence of numerous peculiar phenomena that can occur in a very short period, while the overall environment remains at the ambient atmospheric conditions. After the growth phase, bubbles can collapse, generating new smaller rebound bubbles that can grow and collapse several times. This phenomenon, known as rebound, is due to the presence of gas trapped into the bubbles during collapse [35, ]. Rebound bubbles sometimes can also break into different bubbles (fission of bubbles). The shape of rebound bubbles depends on the symmetry or asymmetry of the collapse dynamics. A perfectly spherical collapse produces a spherical rebound bubble, while an asymmetry leads to the deformation of the bubble and the generation of a micro-jet emerging from the rebound bubble []. A micro-jet can reach velocities in the order of $100 \mathrm{~m} / \mathrm{s}$ [36], increasing the turbulence and the mass transport coefficient. Because of their high velocity, micro-jets can contribute to changes in water clusters, in the agglomeration of fibers and molecules. During the collapse phase, it is possible to reach very high temperatures, in the order of thousands of K [23], but for a very short period (in the order of micro seconds). Under these conditions, the vapours trapped in the cavitation bubbles can be dissociated, with the consequent generation of free radicals, such as ${ }^{\circ} \mathrm{H}$ and ${ }^{\circ} \mathrm{OH}$, which are very strong and non-specific oxidizing species $[37,38]$. Sometimes both the temperature and the pressure into the liquid are so high that light can be emitted during the collapse phase (luminescence [39]).
Cavitation can be further classified, according to the degree of its intensity, which depends on the magnitude of the pressure or temperature, in transient cavitation or stable cavitation [31]. In transient cavitation, the radius of cavitation bubbles expands at least twice its initial size. Thus, cavitation bubbles can collapse to a minute size of its original in a limited period of time, resulting in the absence of mass flow of permanent gas through the liquid-bubble interface [28] and causing a very violent implosion. This gives rise to the release of a significant amount of acoustic energy and the emission of light. In this phase, vapour temperature inside the bubbles can reach several thousand $\mathrm{K}$ and the cavitation pressure several hundred atm. In stable cavitation, on the other hand, cavitation bubbles contain more gas and therefore have a less violent collapse than transitory cavitation bubbles. Shock waves and micro-jets caused by implosion of stable cavitation bubbles usually generate lower pressure than transient ones [40-42].

It has been experimentally proven that in $\mathrm{HC}$, if the intensity of the turbulence is rather low, the recovery pressure downstream of the constriction can be approximated by a linear expression with respect to the distance downstream of the constriction, where the stable cavitation takes place. When the turbulence intensity increases and the recovery pressure is no longer linear, the behaviour of cavitation bubbles can be defined as transient [43].

\section{HC generation and mechanisms}

Due to the introduction into the flow of some constrictions, such as orifice plates, Venturi systems or throttling valves, flow velocities and, consequently, flow pressure can be modified, resulting in $\mathrm{HC}$ generation. According to the Bernoulli principle (Eq. 1), when the liquid flows through a constriction, the flow velocity (Fig. 4 a) and, therefore, the flow kinetic energy increases at the expense of the flow pressure (Fig. 4 b):

$$
\frac{V^{2}}{2 g}+\frac{p}{\rho g}+z=\text { constant }
$$

where $V$ is the velocity of the flow at a point on a streamline, $g$ is the acceleration due to gravity, $z$ is the elevation of the point above a reference plane, $p$ is the pressure at the chosen point, and $\rho$ is the density of the fluid at all points in the fluid.

When the flow accelerates due to the presence of a constriction, the total energy remains constant, but part of this energy is converted into turbulence and heat during the process; therefore, the downstream flow pressure will always be lower than the upstream one. If the restriction throttling is sufficient, the flow pressure at the constriction throat can equal or fall below the vapor pressure of the fluid at the given temperature and vapor cavities can be generated. 


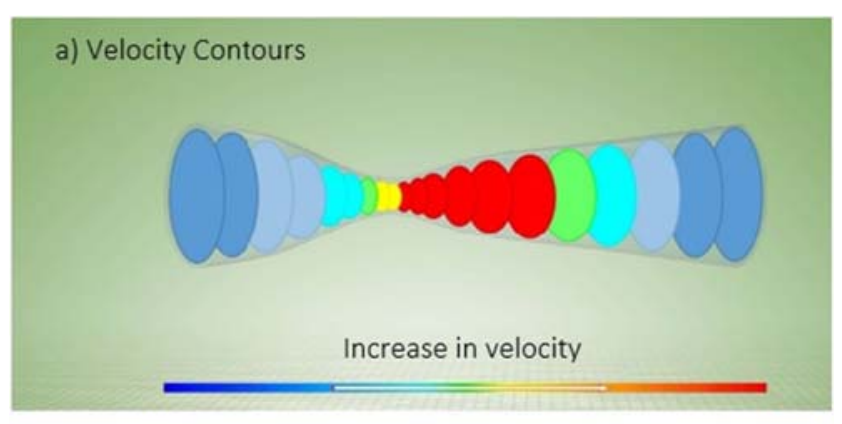

b) Pressure Cortours

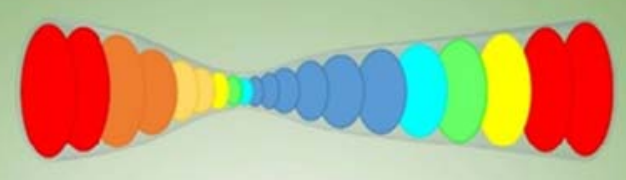

Decrease in pressure

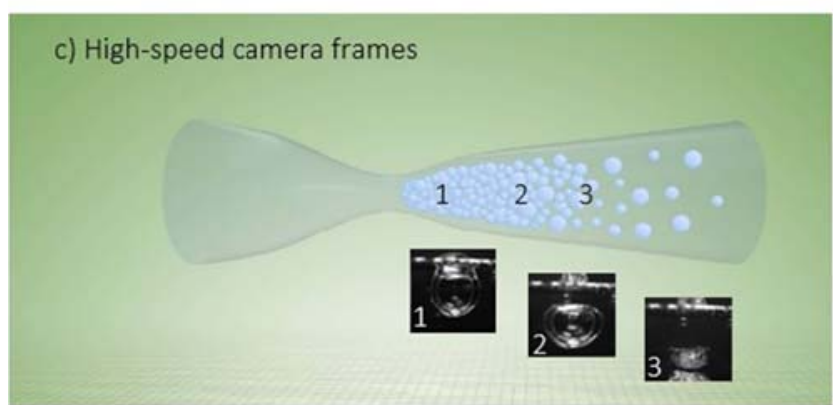

Fig. 4 - Effect of a constriction on flow velocity and pressure in a Venturi system: a) contours of flow velocity magnitude; b) contours of flow pressure magnitude; c) high-speed camera frames. Flow direction is from left to right

This condition can be formulated as follows, Eq. 2:

$p_{\min }=p_{v}$

where $p_{\min }$ is the minimum static flow pressure and $p_{v}$ is the vapor pressure at a given fluid temperature. According to the geometry of the constriction, with further lowering of the pressure, the cavities can continue to grow. They reach their maximum size at the lowest flow pressure and subsequently when the cross section expands, with a consequent decrease in the flow velocity, the pressure flow recovers and this can cause the implosion and the collapse of these previously formed cavities [32, 44], (Fig. 4 c).

\section{HC flow regimes}

In the characterization of cavitating flows, the most used parameter is cavitation number $C_{v}$ It is a dimensionless number, that can be expressed as reported in the following equation, Eq. 3:
$C_{v}=\left(\frac{p_{r-} p_{v(T)}}{\Delta p}\right)=\left(\frac{p_{r-} p_{v(T)}}{\frac{1}{2} \cdot \rho \cdot V^{2}}\right)$

where $p_{r}$ is the recovery pressure $[\mathrm{Pa}]$ measured downstream of the restriction, in which the flow becomes again undisturbed (a conventional reference point can be considered where cavitation inception is expected and the flow pressure is easily measurable), $p_{v}$ the liquid vapour pressure of at the operating temperature $\mathrm{T}$ (i.e. $2.35 \mathrm{kPa}$ at $25^{\circ} \mathrm{C}$ ) [Pa], $\Delta p$ the pressure difference that characterizes the system, and $\rho, V$ are the liquid density $\left[\mathrm{kg} \mathrm{m}^{-3}\right]$ and the flow velocity $\left[\mathrm{m} \mathrm{s}^{-1}\right]$ at the cavitating constriction, respectively.

The cavitational number indicates the probability that cavitation takes place in a flow regime. As shown in Eq. 3, $C_{v}$ is can be defined as the ratio between the forces exerted by collapsing cavities with respect to those initiating their formation. Therefore, high $C_{v}$ values involve more collapsing forces and lower initiating forces, indicating lower cavitational activity. Cavitation may appear, due to a decrease in flow pressure at the reference point or an increase in the $\Delta p$-value.

Cavitation begins to occur for $C_{v}$ values below the threshold value, $C_{v}{ }^{*}$. In most of studies it was observed that the cavities formation started at $C_{v}$ equal to 1 [32]. However, cavitation can also start at $C_{v}$ greater than 1 , because of the presence of dissolved gases into the fluid and other solid particles which can act as pre-nuclei and therefore they can accelerate the phenomena of cavities generation. For example, typical $C_{v}{ }^{*}$ values are ranged from 1.0 to $2.5-2.79,[18$, 45].

Many reports indicate that when a decrease in $C_{v}$ below 1 occurs, more cavities are formed, with consequent increase in cavitational effects. It has also been observed that a further decrease in $C_{v}$ implies the achievement of a condition where the cavities can coalescing with each other, because of the high number and volume concentration of the cavities, forming a vapour cloud (choked cavitation/supercavitation), resulting in a decrease in cavitation intensity [46].

The threshold $C_{v}{ }^{*}$ depends on all the usual factors considered in fluid mechanics, such as flow geometry, viscosity, gravity, surface tension, turbulence levels, thermal parameters, wall roughness and gas content of fluid, in terms of dissolved and free gases [36].

In $\mathrm{HC}$, the comparison between the measured cavitation number and the threshold $C_{v}{ }^{*}$ can lead to the identification of five different flow regimes [36]:

- Non-cavitating flow: when the liquid pressure is higher than the saturated vapour pressure, cavitation does not occur $\left(C_{v}>C_{v}{ }^{*}\right)$. In this flow regime, known as noncavitating flow phase, there is no evidence of cavitation bubbles into the fluid. 
- Cavitation inception or limited cavitation: in this flow regime, the liquid pressure is equal to or lower than the liquid vapor pressure. The cavitation number is equal to or slightly lower than the threshold value $\left(C_{v} \leq C_{v}{ }^{*}\right)$. Cavitation is barely detectable, with the consequent appearance of scattered cavitation bubbles into the fluid. This phase is always characterized by a loud noise of cavitation bubbles that collapse, due to the instability of the generated cavities.

- Developed cavitation: even lower values of the cavitation number $\left(C_{v}<<C_{v}{ }^{*}\right)$ allow the complete development of the cavitation bubbles.

- Supercavitation: this flow regime represents the final state of cavitation $\left(C_{v}<<<C_{v} *\right)$. In this regime, the pressure in the cavitation area is low and a large fixed cavity is formed. Compared to other types of cavitation, the interface of a supercavitation cavity is stable. The cavity remains attached to the constriction and the cavity closure is downstream.

- Desinent cavitation: ending in this flow regime, cavitation bubbles disappear completely, as the pressure of the downstream flow increases. The desinent cavitation is often considered as a threshold between cavitating and noncavitating flows.

Recently, it has been shown that $C_{v}$ cannot be used as a single parameter in providing cavitation conditions and there are broad inconsistencies regarding its determination in previous reports [47]. Furthermore, $C_{v}$ is not always detectable in $\mathrm{HC}$ devices, because it is too difficult to measure the flow velocity or the pressure where cavitation occurs (turbulent flow), especially when the complexity of the cavitating device geometry increases [8].

\section{HC types}

Furthermore, HC can be differently defined, according to different flow configurations. There are two main classification categories: attached cavitation and vortex cavitation [].

\section{Attached cavitation}

The attached cavitation is easily identifiable due to the beginning of a region characterized by of a separate flow filled by steam, which partly remains attached to the solid surface. When the cavity becomes so high that it completely envelops the solid body, supercavitation can occur. If this type of cavitation occurs in the propeller blades or hydrofoils (Fig. 5 a), it is known as "sheet cavitation", while the name of "blade cavitation" can be associated with the attached cavitation occurring in pumps. The attached cavitation can also be detected in bluff bodies (Fig. 5 b).
In case the generated transient cavities are of the same order as the main attached cavity, the attached cavitation is also called "cloud cavitation". Figure 6 shows the formation (Fig. 6 a), the separation (Fig. 6 b) and the collapse (Fig. $6 \mathrm{c}$ ) of a cavitation cloud on a hydrofoil oscillating in pitch. A very common example of imposed fluctuations is the interaction between rotor and stator blades in pumps or turbines. Recently, the developed cavitation has been studied by Petkovšek et al. [48] in a shear-induced HC reactor, in which two facing rotors were spinning in opposite directions: attached cavitation and bubbles shed from the attached cavitation have been observed on the solid surface of the teeth rotors and in the difference between the rotor and the housing. A cavitation cloud was also detected between the aligned grooves.

In order to effectively exploit the positive effect of $\mathrm{HC}$, the developed cavitation must be studied and controlled in $\mathrm{HC}$ reactors. For example, Dular et al. [22], studying a Venturi device, in which attached cavitation occurred [49], showed the progress of the developed cavitation (Fig. 7).

Elements with different shapes and sizes can differently influence the attached cavitation, mainly due to the onset of a pressure difference between the external flow and the inner part of the attached cavitation. As a result, the formation of a stagnation point and different forms of the attached cavitation, due to the different tested cavitation conditions, were observed. All the phases involved in the generation of the attached cavitation, in terms of cavitation inception, cavities reshape and rupture, were repeated periodically (Fig. 7 a). Supercavitation (Fig. 7 b) was also observed in the experiment, because of a decrease in flow pressure and, therefore, to an increase in flow velocity.

\section{Vortex cavitation}

When a linear vortex is generated into the liquid, the static pressure in the core is depressed if compared with the pressure far from the axis of the vortex. When the pressure in the vortex core falls below the liquid vapour pressure, the vortex cavitation can occur. In such situation, the entire vortex core may become filled with vapour. Figure 8 shows some examples of vortex cavitation in a hydrofoil (Fig. 8 a), in a propeller (Fig. 8 b) and in a turbine (Fig. 8 c), respectively. It may happen that vortex cavitation bubbles remain smaller compared to the vortex core radius, with nearly spherical rapidly growing and collapsing bubbles entirely within the confines of the vortex core. However, at lower flow pressures the initially near-spherical bubble can expand and elongate to fill the core of the vortex and continue to grow along the vortex axis, becoming highly elongated [50]. 
Fig. 5 Attached cavitation on: a) hydrofoil and b) bluff body. Flow direction is from left to right (reproduced from Brennen [29])
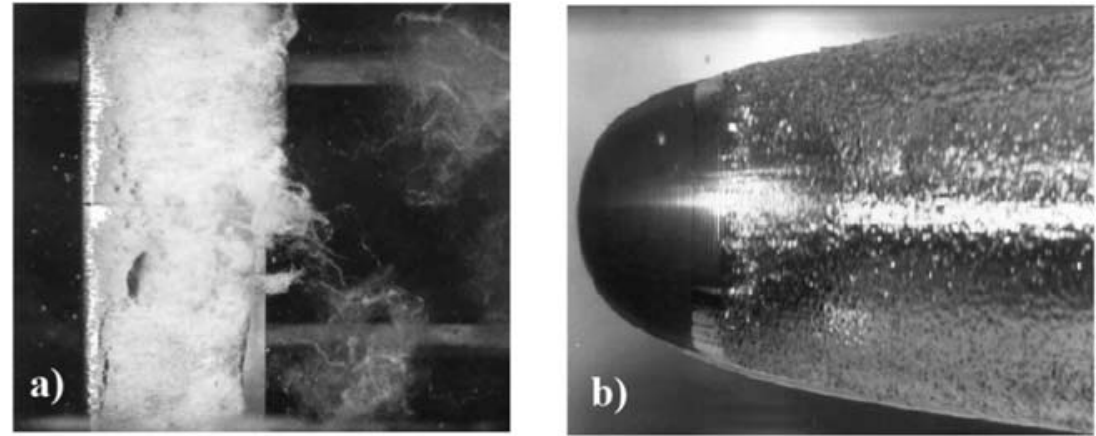

\section{Modeling}

In the last decade, $\mathrm{HC}$ has been deeply studied by many researchers worldwide. As reported in the following sections, it was observed that essential parameters and operating conditions can deeply influence $\mathrm{HC}$ intensity and the way in which it can be generated. The definition and the characterization of these parameters (i.e. flow velocity, flow pressure, etc.) present some difficulties basically due to the presence of turbulent flows in cavitation conditions in addition to the complexity of the geometry of most of the HC devices. Moreover, as highlighted by Šarc et al. [47], these parameters were measured using different procedures by researchers, resulting in large inconsistencies reported in the scientific reports. For this reason, experiments and observed results are often not repeatable. To confirm this, it was observed that flow velocity and flow pressure measurements can be easier made in simpler geometries such as hole orifice plates, nozzles or Venturi systems. On the contrary, in cavitating devices with a more complex geometry, it is rather difficult to perform measurements on these variables, resulting in an incomplete or incorrect characterization of the $\mathrm{HC}$ process.

To overcome these limitations, in the last years there is an increasingly interest in developing mathematical models with the main goal to define a powerful tool able to provide information on hydraulic parameters and on the fluid dynamics into $\mathrm{HC}$ devices. Indeed, many researchers are focusing their attention on mathematical modelling and many attempts have been made in order to build models in which $\mathrm{HC}$ is taken into account. Again, the complexity of the geometry of some HC reactors and the presence of turbulent conditions in the fluid can increase the difficulty in defining the mathematical model.

Simple geometries (i.e. orifice plates, nozzles and Venturi systems) were modelled by Palau-Salvador et al. [51] by performing a computational fluid dynamic (CFD) analysis with FLUENT 6.1 in order to prevent the cavitation inception in the studied cavitational systems. The influence of different geometric parameters such as orifice thickness, hole inlet sharpness and wall angle on the cavitation behaviour was investigated by Simpson and Renade [52] in an orifice device.

Pawar et al. [53] compared four different HC devices characterized by different geometries, observing different behaviours of flow into the devices. In order to calibrate and validate the mathematical model (FLUENT 6.3), their methodology involved also the use of high-speed photographs observations. Navickas and Chen [54] studied the internal flow characteristics of a Venturi system by means of a three-dimensional fluid flow program (FLOW-3D). According to their results, numerical methods were effective in figure out how different parameters may affect the performance of the cavitating device. In order to optimize a multi-hole injector nozzle, He et al. [55] modelled the three-dimensional nature of the flow in the nozzle investigating the effect of the geometry and dynamics factors on the spray characteristics in cavitating conditions. A numerical model was develop by Müller et Kleiser [56] for a vortex breakdown in a non-cavitating flow. Ashrafizadeh and
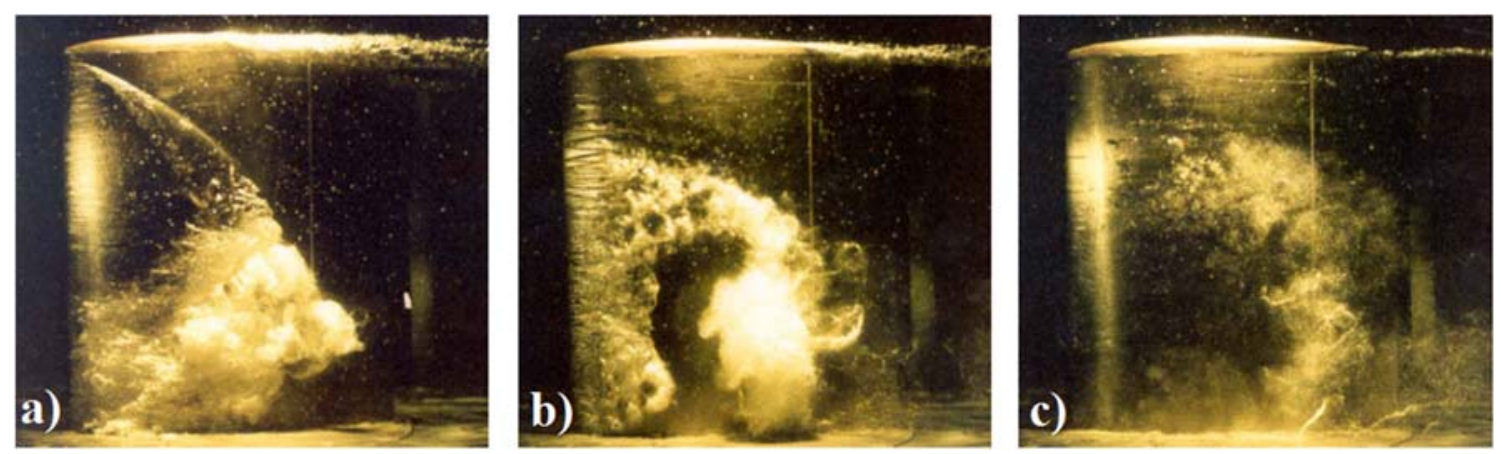

Fig. 6 (a) Formation, b) separation and c) collapse of a cavitation cloud on the suction surface of a hydrofoil. Flow direction is from left to right (reproduced from Brennen [29]) 
(a)

Developed hydrodynamic cavitation
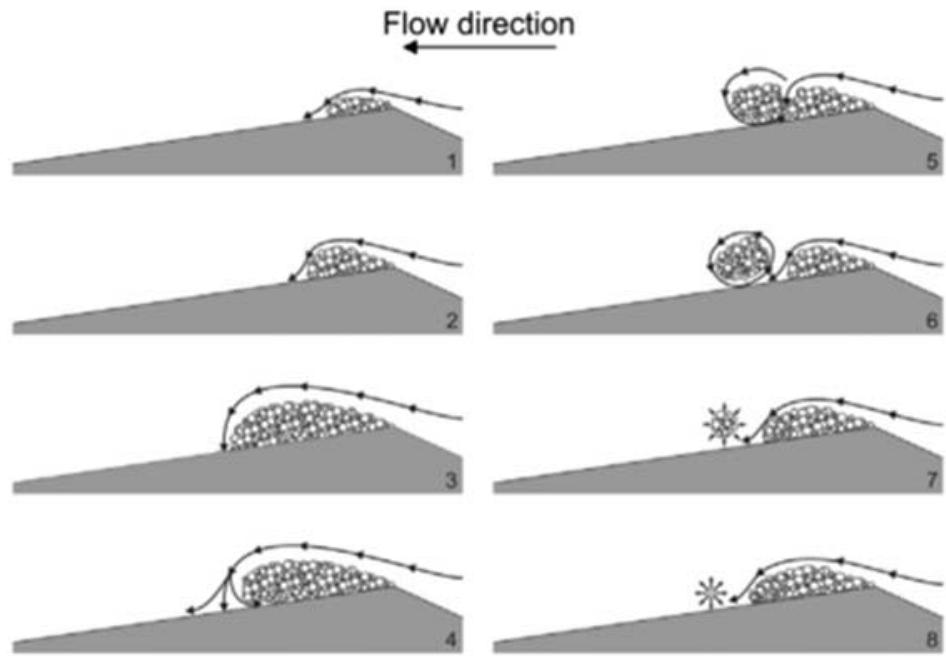

(b)

Supercavitation
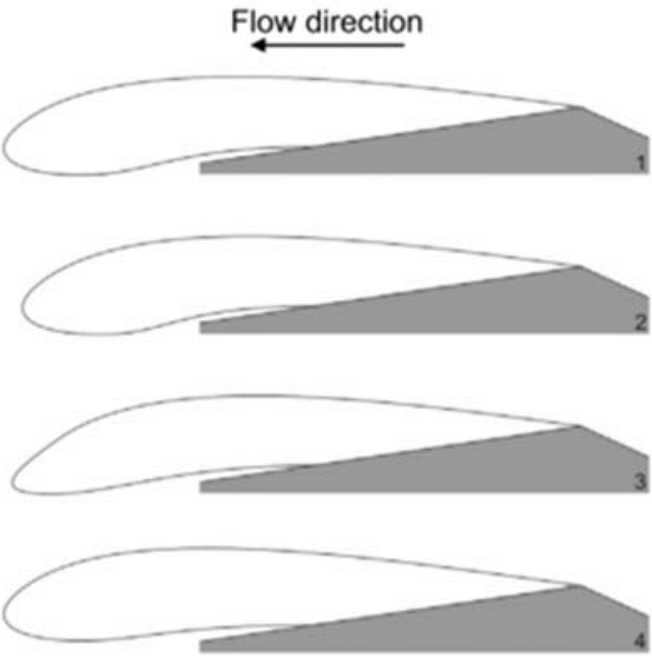

Fig. 7 Schematic representation of a) "developed hydrodynamic cavitation" where highly dynamical vapor cloud shedding associated with high pressure pulsations is expected and b) "supercavitation", which is characterized by a single quasi steady large cavitation pocket (reproduced from Dular, M. et al. [22])

Therefore, an accurate mathematical model could be used as a useful tool in order to optimize parameters, operating conditions and geometry of the cavitating device with the main aim of improving performances of $\mathrm{HC}$ devices in terms of generated cavitation intensities.

\section{Methods to evaluate the efficiency of cavitation}

In the last years, in order to investigate the efficiency of different HC systems in WWTPs in terms of both pollutants removal efficiency and total energy supplied to the system, many methods have been proposed, depending mainly upon the treated matter characteristics.

\section{Pollutants degradation in wastewaters}

$\mathrm{HC}$ technique has recently been used alone or in presence of additives to degrade toxic and carcinogenic compounds in models and the experimental data $[56,57]$.
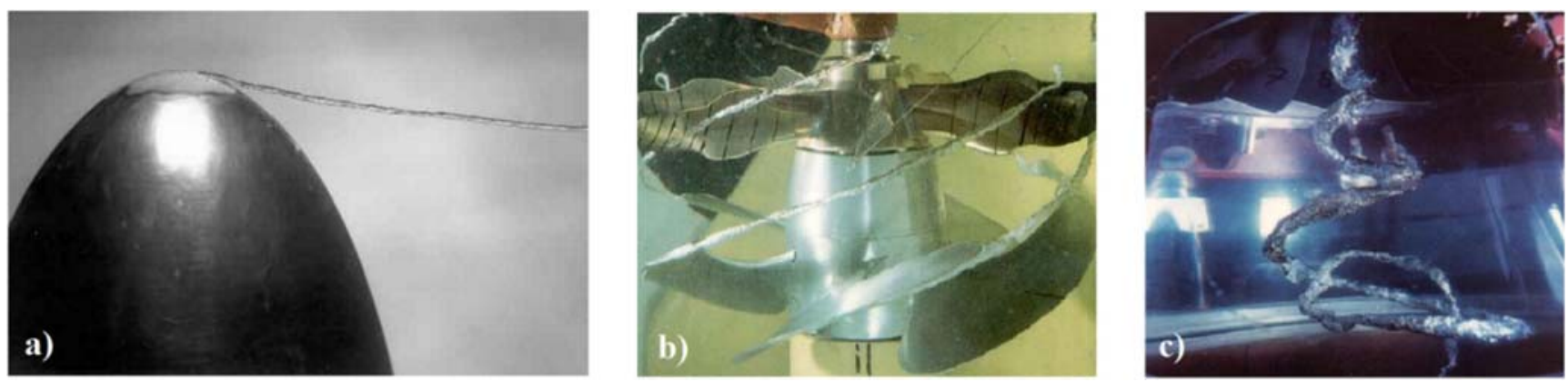

Fig. 8 Vortex cavitation in a) hydrofoil, b) propeller and c) turbine (reproduced from Brennen [29]) 
polluted water bodies. In different studies, the degree of the degradation of pollutants, such as for Rhodamine $\mathrm{B}(\mathrm{RhB})$ and Rhodamine 6G (Rh6G) [8, 14, 60-62], orange 4 dye, orange acid II [63], brilliant green [63], reactive brilliant red K-2BP [64], methylene blue (MB) dye [65], pharmaceuticals [66] and pharmaceutical micro-pollutants $[66,67]$ has been evaluated by considering the extent of degradation (ED), as a percentage of removal. ED can be calculated as reported in Eq. 4:

$E D(\%)=\frac{\left(C_{0}-C\right)}{C_{0}} \cdot 100$

where $c_{0}\left[\mathrm{mg} \mathrm{L}^{-1}\right]$ is the initial pollutant concentration and $c$ $\left[\mathrm{mg} \mathrm{L}^{-1}\right]$ is the residual pollutant concentration at the generic instant. All studies reported that for higher ED, higher HC efficiencies, in terms of extent of pollutant degradation, have been observed.

Another parameter that has been taken into account is the cavitational yield, C.Y. $[8,14,60,63,68,69]$, calculated as reported in Eq. 5, and defined as the ratio of the observed cavitational effect, in terms of amount of degraded pollutant (usually expressed in $\mathrm{m} \mathrm{L}^{-1}$ ) by using $\mathrm{HC}$, to the total energy supplied to the system:

C.Y. $=\left(\frac{\text { Degraded matter }}{\text { Power density }}\right)$

where the degraded matter is the amount of pollutant $\left[\mathrm{mg} \mathrm{L}^{-1}\right]$ removed during the $\mathrm{HC}$ treatment, while the power density, $\left[\mathrm{J} \mathrm{L}^{-1}\right]$, is represented by the following equation Eq. 6 :

Power density $=\frac{P_{a b s} \cdot t}{V}$

where $V$ is the volume of the treated matter [L], $P_{a b s}$ is the pump absorbed power by the HC system [W] and $t$ is the treatment time [sec]. Higher C.Y. values imply higher $\mathrm{HC}$ efficiencies, in terms of both degradation and total energy supplied to the HC system. Nevertheless, C.Y. can be used to compare two or more different $\mathrm{HC}$ systems with each other only if the treated pollutant is the same. On the contrary, treating different contaminants with different $\mathrm{HC}$ systems, it is not possible to make a comparison between their treatment efficiencies because of the different properties and molecular structures of pollutants, which can provide a different resistance to the $\mathrm{HC}$ treatment.

\section{Biological wastewater treatments}

In the field of biological treatments, $\mathrm{HC}$ efficiency can be evaluated by measuring (i) the improvement of solubilisation of the activated sludge in terms of soluble chemical oxygen demand $(S C O D)$ increase, Eq. 7, (ii) the ratio of change in soluble chemical oxygen demand (SCOD) after cavitation to particulate chemical oxygen demand $\left(P C O D_{0}=T C O D\right.$ -
$S C O D_{0}$ ), Eq. 8, and (iii) the ratio of change in ammonia after cavitation to initial organic nitrogen content $\left(\operatorname{Norg}_{0}=T K N_{0}\right.$ $\mathrm{NH}_{4}{ }^{+}-\mathrm{N}, 0$ ), Eq. 9:

$$
\begin{aligned}
& \triangle S C O D(\%)=S C O D_{c a v}-S C O D_{0} \\
& D D_{P C O D}(\%)=\frac{S C O D_{c a v}-S C O D_{0}}{P C O D_{0}} \times 100 \\
& =\frac{S C O D_{c a v}-S C O D_{0}}{T C O D-S C O D_{0}} \times 100 \\
& D D_{N}(\%)=\frac{N H_{4}^{+}-N_{c a v}-N H_{4}^{+}-N_{0}}{N_{o r g}} \times 100 \\
& =\frac{N H_{4}^{+}-N_{c a v}-N H_{4}^{+}-N_{0}}{T K N_{0}-N H_{4}^{+}-N_{0}} \times 100
\end{aligned}
$$

where $S C O D_{c a v}$ is the soluble COD of the treated sludge by using $\mathrm{HC}\left[\mathrm{mg} \mathrm{L}^{-1}\right]$ at the time t, $S C O D_{0}$ is the soluble COD of the untreated sludge $\left[\mathrm{mg} \mathrm{L}^{-1}\right], T C O D$ is the total COD of the untreated sludge $\left[\mathrm{mg} \mathrm{L}^{-1}\right], \mathrm{NH}_{4}{ }^{+}-N_{c a v}$ is the ammonia content of the treated sludge by using $\mathrm{HC}\left[\mathrm{mg} \mathrm{L}^{-1}\right]$ at the time t, $\mathrm{NH}_{4}^{+}-\mathrm{N}_{0}$ and $\mathrm{TKN}$ are the ammonia and total Kjeldahl nitrogen content of the untreated sludge $\left[\mathrm{mg} \mathrm{L}^{-1}\right]$.

Another important index reported in different studies on sludge disintegration is the sludge disintegration degree calculated as the ratio of SCOD-increase by cavitation to the SCOD-increase over the chemical disintegration, Eq. 10:

$D_{\mathrm{COD} \mathrm{NaOH}}(\%)=\frac{S C O D_{\mathrm{Cav}}-S C O D_{0}}{S C O D_{\mathrm{NaOH}}-S C O D_{0}} \times 100$

where $S C O D_{\mathrm{NaOH}}\left[\mathrm{mg} \mathrm{L}^{-1}\right]$ is the soluble COD of the reference sample obtained with a strong alkaline disintegration ( $\mathrm{NaOH}$ digestion).

Furthermore, HC efficiency can be evaluated, according to Eq. 11, as percentage of biogas yield increase by $\mathrm{HC}$ pretreatment [70] [ref]:

$\eta_{C H 4}(\%)=\frac{Y_{\text {biogas with } H C}-Y_{\text {biogas without } H C}}{Y_{\text {biogas without } H C}}$

where $Y_{\text {biogas without } H C}$ is the biogas yield from activated sludge without $\mathrm{HC}$ treatment $\left[\mathrm{ml} \mathrm{gVS}^{-1}\right], Y_{\text {biogas without } H C}$ is the biogas yield from activated sludge with $\mathrm{HC}$ treatment [ml $\left.\mathrm{gVS}^{-1}\right]$.

However, $\mathrm{HC}$ efficiencies should be always referred to other indexes that consider the energy consumption in each $\mathrm{HC}$ system. Many studies report the specific supplied energy $(S E)$, defined as the energy required by the HC system to the initial amount of total solids Eq. 12:

$S E\left(\frac{k J}{k g T S}\right)=\frac{P_{a b s} \times t}{V \times T S}$

where $P_{a b s}$ is the power absorbed from the pump [W], $t$ is the 
treatment time [sec], $V$ is the volume of the treated sludge [L] and $T S$ is the activated sludge solids content $\left[\mathrm{g} \mathrm{L}^{-1}\right]$.

Similarly, the energy efficiency $(E E)$, expressed as $\mathrm{mg}$ DSCOD kJ ${ }^{-1}$, has been calculated as the mg of SCODincrease per unit of energy supplied, Eq. 13, [71]. Higher EE values correspond to higher removal efficiencies.

$E E\left(\frac{m g \Delta S C O D}{k J}\right)=\frac{V \times \Delta S C O D}{P_{a b s} \times t} \times 1000$

\section{The effect of various parameters on HC effectiveness}

A significant number of parameters exist that can affect $\mathrm{HC}$ effectiveness, influencing the number of generated (reactive) bubbles and collapse conditions. As showed in Fig. 9, an overview of the effective parameters in $\mathrm{HC}$ has been suggested by Braeutigam et al. [72].

The main factors that govern $\mathrm{HC}$ and its effectiveness are (i) construction parameters, (ii) technological process parameters of the $\mathrm{HC}$ device, and (iii) properties of the liquid [73]. The influence of all these aspects on HC effectiveness is separately analysed in the following paragraphs.

\section{Construction parameters of cavitating devices}

A first group of factors that influences $\mathrm{HC}$ effectiveness include parameters linked to the structural characteristics of $\mathrm{HC}$ reactors, and more in details of the cavitation inducer and the flow chamber.
Influence of geometry of cavitating devices

HC effectiveness depends on size and shape of cavitation inducer and flow chamber, which can both affect the number of cavitation bubbles and the collapse intensity. Recently, many researchers have focused on the optimization of geometry of cavitating devices to obtain the highest cavitational effects, in terms of both number of cavitation events and significant pressure drops, also resulting in a decrease in energy requirements of the $\mathrm{HC}$ process. With this purpose, different $\mathrm{HC}$ devices have been developed. A first HC reactors classification has been provided by Gogate et al. [74], and successively reported by Manickam and Ashokkumar [75], and adapted in this review:

a) high pressure hydrodynamic cavitation reactor, which is a high pressure positive pump with a throttling device [74] or a blade [76] designed to operate at high pressure ranging from $50 \mathrm{~atm}$ to as high as $300 \mathrm{~atm}$;

b) low pressure hydrodynamic cavitation reactor, where the flow through the main line is forced to pass through a geometric constriction, such as single-hole orifice or multiple holes on an orifice plate [8,72] (Fig. 10 a), a Venturi [16, 46, 47] (Fig. 10 b), a blade, and where the local velocities suddenly increase due to a reduction in the flow area resulting in lower pressures;

c) low pressure swirling cavitation reactor, where cavitation is generated by using a vortex flow swirl chamber [77] (Fig. $10 \mathrm{c}$ ) in which a central vortex with a core pressure lower than the vapour pressure of the liquid is formed, thus inducing cavitation bubbles formation in the vortex, and where the liquid is then ejected from the chamber

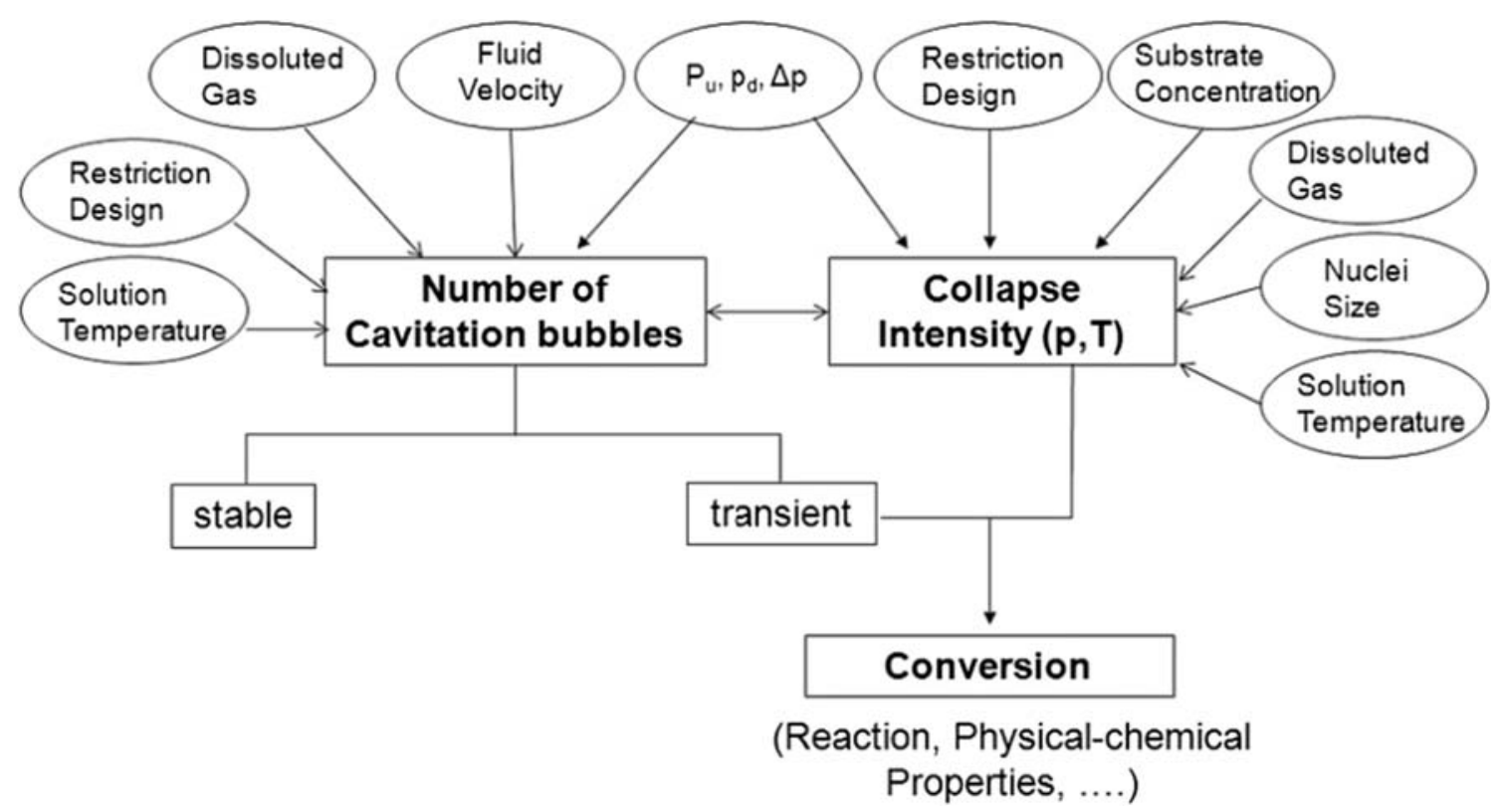

Fig. 9 Overview of the effective parameters in hydrodynamic cavitation (reproduced from Braeutigam, P. et al. [72]) 
through the exit orifice into a volume of liquid where the cavitation bubbles collapse;

d) high speed homogenizer, which consist of rotating equipment (Fig. $10 \mathrm{~d}$ ). Generally a roto-stator assembly is used [19], that operates at rotating speeds of $4000 \mathrm{rpm}$ to as high as $20,000 \mathrm{rpm}$, causing a pressure drop near the periphery of the rotor.

In the first three groups the passage of the liquid supplies the energy needed to induce cavitation (static hydrodynamic cavitation devices) while in the fourth group the energy required is furnished by the help of a rotating inducer (dynamic hydrodynamic cavitation devices).

Further, different geometrical configurations of each device have been investigated in wastewater treatment field. Following are discussed some examples of different $\mathrm{HC}$ reactors reported in literature.

Several researchers investigated the influence of the geometry of orifice plate systems on $\mathrm{HC}$ intensity $[14,78]$, testing plates with different thickness $[8,78]$ and with different numbers, shapes and sizes of holes $[14,78,79]$. It was observed that the highest cavitation intensity was generated by using the lowest free area in the orifice plate system. However, keeping constant the free area, orifice plates with higher diameter and lower number of holes showed lower intensities $[12,14]$.

Šarc et al. [47] compared four very similar Venturi shapes, considering the same operating conditions (constant values for flow pressure, flow velocity, and temperature, respectively). Significant differences in typology, dynamics and intensity of cavitation were found. By using a high-speed camera, they observed how cavitation size and appearance could vary significantly when the divergence angle of the Venturi system was changed. Similarly, Kim et al. [16] investigated the effect of different Venturi outlet inclination angles on the performance of their $\mathrm{HC}$ system, which was used to increase sludge solubilisation. Rajoriya et al. [80] used a slit and a circular Venturi, respectively, to generate cavitation and, thus, to degrade Rhodamine $6 \mathrm{G}$. Considering the same operating conditions, the degradation rate of the dye was marginally higher in the case of slit Venturi as compared to the circular one. In another study [81], the authors checked the influence of geometrical parameters on decolorization of reactive blue 13 in a polluted aqueous solution. With this purpose, eight cavitating

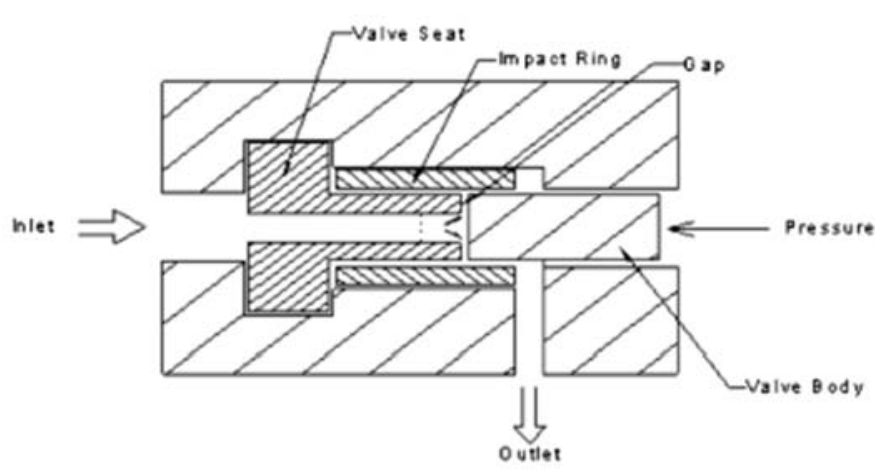

a)

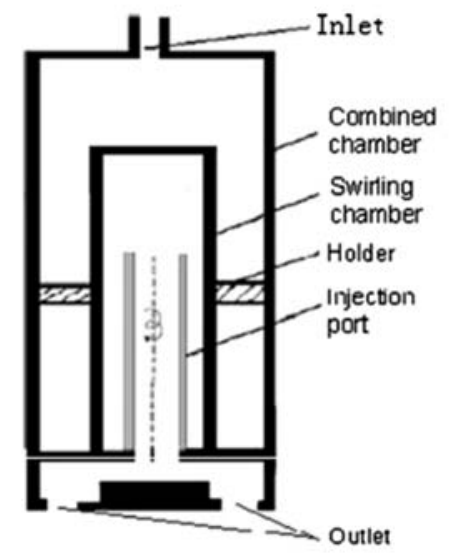

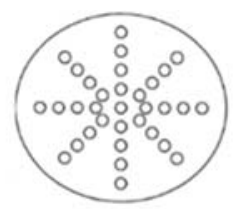

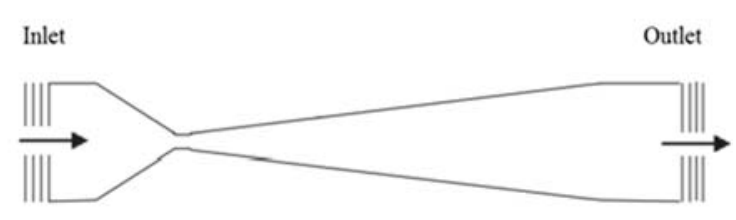

b)

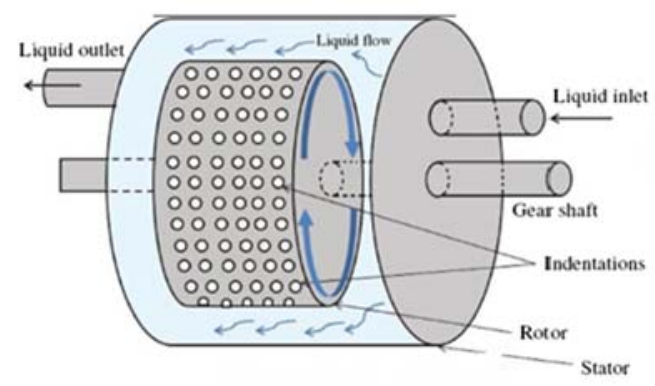

d) 
devices i.e. six orifice plates having different perimeter and flow area, and slit and circular Venturi were tested, showing that the $\mathrm{HC}$ efficiency was strongly influenced by the geometrical parameters. Carpenter et al. [82] provided a detailed geometrical analysis of $\mathrm{HC}$ devices in order to investigate the effect of geometry of orifice plates and Venturi of different shapes and geometrical parameters on the formation and stability of mustard oil in water nanoemulsion.

A swirling jet cavitation device has been investigated by Wang et al. [60, 77]. Recently, Mancuso et al. [8,9] and Langone et al. [83] proposed a modified swirling jet-induced cavitation reactor, in which cavitation was generated by using a modified Ventury system. The authors studied the influence of the injection slots number, the geometry of the system, and the number of $\mathrm{HC}$ devices in series on the $\mathrm{HC}$ effectiveness, highlighting how different geometries of the cavitating device influenced the $\mathrm{HC}$ effectiveness. A comparison between orifice plates and a vortex diode system on degradation of different organic solvents such as acetone, methyl ethyl ketone, and toluene was carried out by Suryawanshi et al. [84].

Badve et al. [85] and Patil et al. [20] developed an efficient stator and rotor assembly, where rotor is a solid cylinder which has indentations on its surface, and can be rotated at different speeds of rotations. Afterwards, a novel stator and rotor configuration has been proposed by Petkovšek et al. [19] based on two facing rotors with special radial grooves spinning in the opposite direction: the authors studied the effect of different shapes of the teeth of their rotor on the cavitation process [48]. Another reactor, which can generate shear cavitation, was recently proposed by Petkovšek et al. [19]. The main feature of this device is that it works both as cavitation generator and as driving pump, avoiding additional pressure drop. Jain et al. [86] studied the influence of the reactor geometry by comparing an $\mathrm{HC}$ rotating device with an orifice plate system for water disinfection.

\section{Technological process parameters}

This second group includes operative parameters of the technological process, such as flow velocity or kinetic energy of the liquid, pressure at the inlet to the $\mathrm{HC}$ system, temperature of the liquid, $\mathrm{pH}$ of the liquid, and the HC "processing" time (the number of times the liquid passes through the cavitation device).

\section{Influence of flow velocity}

In an $\mathrm{HC}$ device, the number of cavitation bubbles is mainly related to an increase of the flow velocity or the kinetic energy of the liquid. Increasing the flow velocity, higher local pressure oscillations are expected, and pressure may fall below the vapour pressure of the liquid at the operating temperature, causing partial vaporization of the liquid and the generation of cavities. As can be deduced from Eq. 3, an increase in flow velocity implies a decrease in the cavitation number, resulting in an increase of cavitational effects. Higher flow velocities further can imply higher turbulence levels.

Flow velocity can be varied either by adjusting the pump flow rate (and thus the pressure at the outlet of the pump) or by changing the geometry of the cavitating device (i.e. reducing or increasing the constriction area).

Šarc et al. [47] reproduced different cavitation conditions by varying the flow velocity at the throat of a Venturi system for different values of inlet pressure, while keeping constant the cavitation number and the geometry of their HC system. They observed that cavities slightly grew when the flow velocity was increased.

A Venturi system was used by Saharan et al. [46] to investigate the effect of the flow velocity on degradation of RR120. It was observed that the degradation rate increased with an increase in the flow velocity reaching a maximum and then it dropped, due to the onset of the supercavitation. Similar results were reported by Prajapat and Gogate [87] and Gore et al. [69] using conventional Venturi systems.

Similarly, Joshi and Gogate [88] investigated the influence of flow velocity at the throat of a single orifice plate by adjusting the flow rate. In their study, increasing the flow velocity up to an optimum value, an increase in the extend of degradation of dichlorvos was detected. Higher flow velocities promoted an increasingly violent collapse of cavities, and thus an increasingly hydroxyl ions generation, resulting in an increment in the extent of degradation. However, for flow velocities higher than the optimum value, the extent of degradation decreased due to the onset of supercavitation, resulting in an extremely rapid growth of bubbles downstream to the orifice plate with the subsequent splashing and vaporization of the flow.

Wang and Zhang [89] reported a continuous increase in the degradation rate of alachlor by using a swirling jet-induced reactor and increasing the flow, while keeping constant the geometry of their $\mathrm{HC}$ system. These results are in agreement with the study of Mancuso et al. [9] that, studying a modified swirling jet-induced cavitation reactor to improve the sludge disintegration, observed an increase in sludge solubilisation with an increase of the flow velocity into the liquid. However, using the same $\mathrm{HC}$ device to treat a polluted dye aqueous solution by Rhodamine B, supercavitation occurred and an optimum value for flow velocity was detected [16].

In a rotor-stator system, Badve et al. [58] varied the velocity of the liquid on the surface of the rotor by adjusting the rotational speed of the rotor. Their results showed that an increase in the rotational speed led to an increase in velocities of the liquid surface and to a decrease of the cavitation number.

Nevertheless, also the geometry can deeply influence the flow velocity, resulting in different cavitational effects. 
Sivakumar and Pandit [14], using multiple hole orifice plates and operating at the same inlet pressure, observed an increase in the cavitation process efficiency increasing the flow velocity for different geometries of their HC system. By using a swirling jet-induced reactor, Mancuso et al. [9] obtained an increase in $\mathrm{HC}$ effectiveness, in terms of activated sludge solubilisation, for the highest flow velocity achieved by decreasing the number of the injection slots at the same inlet pressure.

\section{Influence of inlet pressure to the HC system}

Inlet pressure can influence both the number of generated cavitation bubbles and their collapse intensity. In HC systems, an increase in inlet pressure always implies an increase in flow rate, and vice versa. As seen in section 6.1.1, higher inlet pressures involve higher flow rates and thus higher flow velocities, increasing turbulence levels and local pressure oscillations. Therefore, increasing the inlet pressure an enhancement of $\mathrm{HC}$ effectiveness is expected. Furthermore, higher inlet pressures involve higher pressure drops through $\mathrm{HC}$ devices. This increase in pressure can lead to higher shear forces that are able, especially for HC applications in WWTPs, to break down bacterial cell walls and to increase the release of organic matter $[9,90]$. However, at very high inlet pressures cavities can start coalescing with each other and supercavitation can be detected, resulting in a progressive decrease in the $\mathrm{HC}$ effectiveness. By using both a multi-hole orifice plate system and a swirling jet reactor, Mancuso et al. [8] observed that higher inlet pressures allowed to achieve higher extent of degradation rates in polluted aqueous solutions containing Rhodamine B. Similarly, Kumar and Pandit [91] found an increase in the pressure drop across their Venturi system due to higher inlet pressures to the HC system, resulting in an increase in the cluster collapse pressure. Two different systems (slit and circular) were used by Rajoriya et al. [80] to treated wastewater polluted by Rhodamine 6G. The Authors observed an increase in the extent of degradation by increasing the inlet pressure up to an optimum value of 5 bar. However, lower decolourization rates were detected by considering inlet pressures beyond 5 bar, due to the inception of choked cavitation.

Similar findings were reported from Kumar et al. [65] that treated polluted aqueous solution by methylene blue dye by means of a Venturi system. In their experiments, the inlet pressure was varied from 1 to 10 bar, showing an optimum at 5 bar in terms of extent of dye degradation. Thanekar et al. [92] investigated the effect of inlet pressure on degradation of carbamazepine. The cavitational intensity increased with an increase in pressure from 3 bar until an optimum of 4 bar. Further increase in pressure beyond optimum value led to the formation of cavity cloud which in turn reduced cavitational intensity, resulting in a decrease in the extent of degradation. Carpenter et al. [82] used two Venturi systems and single orifice plates with different shapes in order to produce highly stable oil in water emulsion. For all devices, increasing the inlet pressure from 5 to $10 \mathrm{bar}$, a significant increase in $\mathrm{HC}$ efficiency was observed by the authors, but further increasing the pressure from 10 to $15 \mathrm{bar}$, only a marginal or no further increase in HC efficiency was observed. Choi et al. [93] tested different inlet pressure from 30 to 70 bar for the degradation of bisphenol A. Again, the degradation rate increased from 30 to an optimum of 50 bar, then, for a further increase in the inlet pressure, the downstream area into the Venturi system was completely filled with cavities, which began to coalesce with each other and formed a cavity cloud, resulting in a decrease in the extend of degradation. The effect of inlet pressure on the degradation of Rhodamine B was investigated by Tao et al. [94] over the range of 60-120 bar. An increasing degradation rate for Rhodamine B at higher inlet pressure was observed from the authors. In this case, supercavitation was not detected simply because inlet pressure was not high enough or most likely due to the capacity of jets impingement to divide cavitation bubbles into micro-bubbles and then to prevent coalescence between them.

\section{Influence of cavitation number (Cv)}

Cavitation number, $C v$, is inter-correlated with the other two parameters previously described: flow velocity and inlet pressure. Up to now, many studies on the influence of $C v$ on the effectiveness of cavitation have been carried out. However, missing or inaccurate information about the position at which the pressure and the flow velocity were measured or calculated implies that researchers commonly use inappropriate values of $C v$, taking advantage that there are no standard methods for its determination [47]. Most of the studies on $\mathrm{HC}$ are not repeatable because of a not detailed definition of the $C v$ in the published research. Nevertheless, for completeness of the contents of this work, some results on the influence of $C v$ on the $\mathrm{HC}$ effectiveness have been reported.

Tao et al. [33] recently investigated the effect of the $C v$ on degradation of Rhodamine $\mathrm{B}$ by using a novel device based on double cavitating-jets impingement. A decrease in the $C v$ from 0.0486 to 0.0246 implied an increase in Rhodamine B degradation. However, in their results the authors reported that the pressure in the main chamber was just approximatively 10 bar. This inaccuracy was due to the difficulty to get an accurate value of the pressure, and hence of the $C v$ (see definition of this parameter above in Eq. 3), most likely due to the presence of cavitational conditions that made measurements more difficult. As expected, Rajoriya et al. [81] observed a decrease in $C v$ with an increase of the inlet pressure for different geometries of their $\mathrm{HC}$ system in order to treat a polluted solution by reactive blue 13. In their study, it was found that the decolorization rate increased with a decrease 
in $C v$ till an optimum value and then further decreased or remained constant for all the cavitating devices used, due to the inception of chocked cavitation. Saharan et al. [46] used a Venturi system to degrade Reactive Red 120 dye. It was observed that the rate of degradation increased with a reduction in $C v$, reaching a maximum and then dropping. In their findings, authors distinguished two different stages depending on cavitation number: firstly, a decrease in cavitation number led to an increase in the number of generated cavitation bubbles, resulting in an increase in the number of cavitational collapse pressure pulses as well as in the generation of more ${ }^{\circ} \mathrm{OH}$ radicals that increased the degradation rate; then, the degradation rate decreased with further decrease in $C v$. This may be because of reduced cavitational intensity due to excess numbers of cavities inside the Venturi system at very low $C v$. Indeed, when there are too much cavities, these can start coalescing to form a larger cavitational bubble that can get away from the liquid without collapsing or result into an incomplete collapse, resulting in a reduction of generated ${ }^{\circ} \mathrm{OH}$ radicals.

Gore et al. [69] investigated the influence of the $C v$, ranged from 0.210 to 0.095 , on degradation of reactive orange 4 dye using a Venturi system. Also in this case, it has been found that the decolourisation rate increased with a decrease in the $C v$, reaching to the maximum at 0.15 and then decreased. Authors attributed these findings to an increase in the number of cavities and thus of the number of collapsing events, resulting in the generation of more ${ }^{\circ} \mathrm{OH}$ radicals. However, a further decrease in the $C v$ below 0.15 implied a decrease in the extent of degradation due to the occurrence of choked cavitation/ supercavitation conditions, where cavities no longer behaved as individual cavities. They coalesced with each other to form larger vaporous bubbles, which were carried away with the flowing liquid without collapsing.

All studies on wastewaters contaminated by soluble pollutants confirm that for a decrease in $C v$, an increase in the number of cavities is expected, resulting in an increase in overall collapse intensity of cavities [46, 69, 81]. However, depending on the specific $\mathrm{HC}$ device configurations, after certain value of $C v$, the number of cavities can become very high, so these cavities can start coalescing with each other and form a cavity cloud [46, 69, 81], compromising HC effectiveness. Energy produced by the collapse of some cavities can be taken up by the neighbouring cavities, resulting in a cavity cloud formation (choked cavitation). Hence, the importance of optimizing $\mathrm{HC}$ cavitation devices in order to operate between these two limits, i.e. cavitation inception and choked cavitation/ supercavitation, to get the maximum effect.

By applying $\mathrm{HC}$ treatment to sludge, $C v$ greater than 1 have been reported, because of the presence of solid particles into the flow which can act as pre-nuclei and therefore they can accelerate the phenomena of cavities generation. Lee and Han [18] estimated a cavitation number of 2.79 using a multipleorefice plate to treat activated sludge for methane production.
However, very few studies reported $C v$ values when sludge is treated by $\mathrm{HC}$, mainly because of the difficulty to evaluate the various parameters. Further, it should be noted that in order to compare two or more $\mathrm{HC}$ systems each other, rather than $C v$, other parameters, such as for instance vibrations and noise measurements, and some indexes (see section 5) could be used to measure the intensity and efficiency of HC systems.

\section{Influence of liquid temperature}

The influence of temperature on $\mathrm{HC}$ is complex, which can have both positive and negative effects on $\mathrm{HC}$ treatment efficiency. Temperature affects the dynamics of cavities through the properties of the liquid such as viscosity, vapor pressure, and surface tension. As shown in the phase diagram of water (Fig. 3), the vapour pressure of a fluid depends on its temperature and it increases exponentially with temperature. Thus, an increase in operating temperature can both enhance the probability of vaporous cavities generation due to entrapment of vapours and influence the kinetic rate. The increase of temperature implies a decrease of the viscosity and surface tension, leading to an easier bubble formation [73]. However, these generated bubbles are richer in vapour content, which reduce the intensity of bubbles collapse due to the "cushioning" effect of their implosion [73]. A rise in temperature reduces gas solubility, which is the chief source of cavity nuclei and thus reduces the rate of occurrence of cavitational events.

It is then likely that an optimum operating temperature might exist in specific conditions. When the temperature of the solution increases beyond the optimum value, the increase in vapor pressure of liquid might result in higher vapor content in cavitation bubbles leading to a cushioning effect and thus to an $\mathrm{HC}$ effectiveness reduction.

With this background, Wang and Zhang [89] have reported a positive effect on the degradation of alachlor with an increase in temperature from 30 to $40^{\circ} \mathrm{C}$, but the degradation rate decreased with a further increase in the temperature over a range of $40-60{ }^{\circ} \mathrm{C}$. Joshi and Gogate [88] investigated the effect of operating temperature over the range of $31-39{ }^{\circ} \mathrm{C}$. In their experiments on degradation of dichlorvos, the maximum degradation rate was achieved at the lowest temperature. Mancuso et al. [9] investigated the influence of temperature, ranged from 20 to $35^{\circ} \mathrm{C}$, on sludge disintegration. The authors observed that for this range of temperature, the higher the temperature, the more efficient $\mathrm{HC}$ (in terms of sludge solubilisation) was. Similar results were reported by Choi et al. [93] for the degradation of bisphenol A by using HC, where an increasing temperature from 10 to $50^{\circ} \mathrm{C}$ implied an increase in the extend of degradation. Šarc et al. [47] proved that the magnitude of pressure oscillations, which occurs due to cavitation bubbles collapse, increased for temperatures up to $40{ }^{\circ} \mathrm{C}$, but then dropped significantly for higher temperatures. 


\section{Influence of liquid pH}

Typically, acidic or basic conditions are favourable to the degradation of chemical pollutants using $\mathrm{HC}$ reactors. The optimal $\mathrm{pH}$ value depends on the pollutants to be treated. Their degradation is strongly dependent on medium $\mathrm{pH}$, as $\mathrm{pH}$ can influence the presence of ${ }^{\circ} \mathrm{OH}$ free radicals available for pollutants removal. Moreover, it is possible to get changes in pollutants structure for different $\mathrm{pH}$, obtaining more biodegradable forms, which can be easily attacked by ${ }^{\circ} \mathrm{OH}$ free radicals. Usually, acid conditions are recommended for pollutants degradation by $\mathrm{HC}$, because such conditions favours generation of hydroxyl radicals and impedes any reaction of recombination among free radicals [69].

Many studies have been carried out to remove dye pollutants from wastewaters. Saharan et al. [46] investigated the effect of $\mathrm{pH}$ on Reactive Red 120 dye degradation by carrying out experiments at different $\mathrm{pH}$ ranged from 2 to 11 . Their results indicated that the rate of degradation increased with a decrease in the $\mathrm{pH}$ of the solution, confirming that acid conditions implied higher degradation rates for the Reactive Red 120 dye. Also Kumar et al. [65] observed a decrease in the rate of decolourization of polluted solution by methylene blue dye with an increase in the $\mathrm{pH}$ from 2 to 10 , indicating that also the state of the molecules of this dye can change from ionic to molecular or vice versa depending on the solution $\mathrm{pH}$ and thus different rates of decolourization can be detected. These findings are in agreement with results of Rajoriya et al. [81], where the authors investigated the influence of $\mathrm{pH}$ solution, ranged from 2 to 8 , on decolourization of reactive blue 13 dye by using a slit Venturi to generate cavitation. Their results depicted that the extent of decolorization increased with a decrease in solution $\mathrm{pH}$. Gore et al. [69] studied the effect of $\mathrm{pH}$, ranged from 2 to 10 , on degradation of reactive orange 4 dye using a Venturi system. The authors, starting from a neutral polluted solution, observed an increase in the decolourisation rate by either increasing or decreasing the $\mathrm{pH}$ of the polluted solution. However, the highest extent of degradation was obtained working at lower $\mathrm{pH}$ solutions, indicating a lower rate of recombination among generated ${ }^{\circ} \mathrm{OH}$ radicals in acid conditions and, hence, the presence of more 'OH radicals available for the dye oxidation. Moreover, the increase in the degradation rate can be also attributed to the change of state of the orange 4 molecules from ionic to molecular under acidic conditions. This alteration in the state of the molecules makes them hydrophobic and more easily attacked by ${ }^{\circ} \mathrm{OH}$ free radicals. On the contrary, in the basic medium, the extent of degradation of orange 4 was not so high as in acid conditions because dye molecules remained in ionic state, resulting in an increase in their hydrophilic behaviour that implied their persistence in the liquid bulk.

Same findings have been reported by some researchers that studied the degradation of Rhodamine B. Mancuso et al. [8] treated polluted aqueous solutions by Rhodamine B using a multi hole orifice plate system, and observed the highest degradation rate of the dye at $\mathrm{pH} 2$, while it dropped significantly and remained constant for $\mathrm{pH}$ ranged from 5 to 8 . This was due to the prevalence in the acid conditions of Rhodamine $\mathrm{B}$ in the cationic form rather than the zwitterionic form [95]. Previous studies on Rhodamine B reported that the cationic form is easier to degrade [60], and thus at low values of $\mathrm{pH}$ the oxidation of Rhodamine B can be higher. Similar results were obtained by Mishra and Gogate [62] using a Venturi device, by Wang et al. [60] using a swirling jet-induced cavitation system, and by Tao et al. [94] using a novel reactor based on double-cavitating-jets impingement, respectively. A different trend of results on the effect of the $\mathrm{pH}$ was reported by Rajoriya et al. [80], studying Rhodamine $6 \mathrm{G}$ degradation. In this case, the observed results showed an increase in the extent of degradation with a $\mathrm{pH}$ increase in the solution from 2 up to an optimum value of 10 . Then, the decolorization rate decreased. Under acidic $\mathrm{pH}$ Rhodamine $6 \mathrm{G}$ molecule became hydrophilic in nature, and thus, the dye remained in bulk of the solution where concentration of ${ }^{\circ} \mathrm{OH}$ radicals was minimum. On the other hand, under basic conditions Rhodamine 6G molecule became hydrophobic. Under such conditions, the dye was in its molecular state and located itself at the cavity-water interface where the concentration of ${ }^{\circ} \mathrm{OH}$ radicals was maximum. Recently, $\mathrm{HC}$ has been applied to treat water solutions contaminated with insecticide and pharmaceutical compounds, and the $\mathrm{pH}$ effect on pollutant degradation have been also investigated. Joshi and Gogate [88] investigated the influence of $\mathrm{pH}$ on degradation of dichlorvos, a household and public health fumigant. Using an orifice plate system as cavitating device, they found that lower $\mathrm{pH}$ provided higher extents of degradation. Studying the degradation of pharmaceutical compounds, Thanekar et al. [92] found that the efficiency of carbamazepine removal was maximum for a $\mathrm{pH}$ of 4 and minimum at 11 . However, a decrease from 4 to 3 showed a worsening of the degradation process.

When treating activated sludge, it was observed that alkaline conditions were more suitable to enhance organic hydrolysis [96]. Optimal results in terms of organic matter release have been obtained when $\mathrm{HC}$ was assisted with $\mathrm{NaOH}$ pretreatment, then working at basic conditions with a $\mathrm{pH}$ of about 9 or 10, resulting in an increase in biogas production in the anaerobic digestion process [69].

\section{HC schemes}

In order to treat wastewaters, different $\mathrm{HC}$ experimental schemes have been proposed. Usually, $\mathrm{HC}$ devices require low amounts of supplied energy to work and, therefore, they are generally applied to closed loop circuits, where the liquid to be treated is recirculated several times through the $\mathrm{HC}$ reactors (Fig. 11) [8, 14, 22]. Figure 11 shows an example 


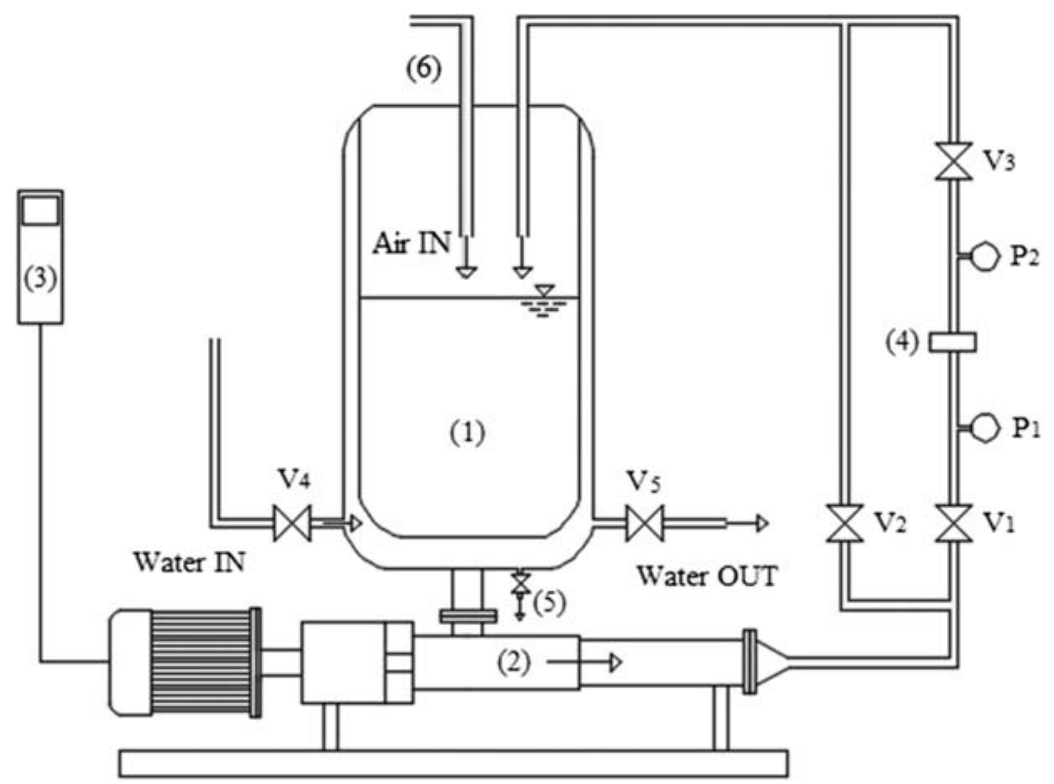

\section{Reservoir \\ 2. Pump \\ 3. Inverter \\ 4. Cavitation reactor \\ 5. Sampling port \\ 6. Compressed air system}

$V_{1}, V_{2}, V_{3}$ Control valves

$V_{4}$, V5 Cooling system valves

$\mathrm{P}_{1}, \mathrm{P}_{2}$ Pressure gauges

Fig. 11 Schematic representation of the experimental setup of a closed loop circuit (adopted from Mancuso, G. et al. [8], Sivakumar, M. and Pandit, A.B. [14], Dular, M. et al. [22])

of a closed loop circuit where $\mathrm{HC}$ has been taken into account. This scheme comprises a main tank (1), a pump (2), control valves at appropriate places $\left(\mathrm{V}_{1}, \mathrm{~V}_{2}, \mathrm{~V}_{3}, \mathrm{~V}_{4}, \mathrm{~V}_{5}\right)$, a cavitation reactor (4), sampling ports (5) and gauges $\left(\mathrm{P}_{1}, \mathrm{P}_{2}\right)$ to measure hydraulic parameters. Control valves $\left(\mathrm{V}_{1}, \mathrm{~V}_{2}, \mathrm{~V}_{3}\right)$ are placed in order to control the flow rate through the mainline.

However, HC closed loop circuits cannot be considered into scaling-up actions in real WWTPs due to the higher volumes of liquid to be treated, leading to the application of other configurations called "one-shot treatment" [97]. In these configurations, $\mathrm{HC}$ treatment is applied as single unit of the WWTP, and it takes place with a single pass without any recirculation of the solution to be treated. However, these $\mathrm{HC}$ schemes can be implemented when the $\mathrm{HC}$ devices are able to transfer high power during only one passage, resulting in the effectiveness of the $\mathrm{HC}$ process.

About the closed loop circuits, both the $\mathrm{HC}$ efficiency and the extent of degradation can significantly increase with an increase in the number of passages through the $\mathrm{HC}$ reactors $[8,98,99]$. It is substantially due to longer exposure times of pollutants to $\mathrm{HC}$ conditions [69]. If the inlet pressure to the $\mathrm{HC}$ system is constant, the number of passes is directly correlated with the process treatment time.

These considerations were confirmed by Save et al. [100] that used an $\mathrm{HC}$ reactor based on a throttling valves system to disrupt cells. Their results showed as an increase in the number of passes and, then, of the time of treatment, led to a corresponding increase in the extent of cell disruption. However, at low inlet pressures, the effect of the number of passes was marginal, but was slowly increased at higher inlet pressures.

\section{Properties of the liquid medium}

This last group includes parameters characterizing the properties of the liquid medium, mainly pollutant concentration, viscosity of the liquid, surface tension and dissolved gas content.

\section{Influence of pollutant concentration}

When cavitation conditions are not varied, the amount of ${ }^{\circ} \mathrm{OH}$ radicals produced in the $\mathrm{HC}$ system should be constant as well. Therefore, for an increase in initial pollutant concentration, a decrease in pollutant removal rate is expected as hydroxyl radicals may not be sufficient to degrade the pollutant completely.

This is true in the case of removal of chemical pollutants dissolved in water solutions. Parsa et Zonouzian [78] studied the effect of the initial dye concentration on degradation of Rhodamine B by using a submerged multi hole orifice plate system. In their experiments, the initial dye concentration was ranged from 2 to $14 \mathrm{mg} \mathrm{L}^{-1}$. They found that the efficiency of the process was inversely proportional to the initial dye concentration. In justifying this result, the authors explained that it might be imputed to an increase in the total amount of dye molecules, while the total amount of free hydroxyl radicals remained constant. These findings are in agreement with other studies on degradation of Rhodamine B [8, 60, 61, 94], Rhodamine 6G [80], reactive blue 13 [81], tetracycline [101], and other organic solvents such as acetone, methyl ethyl ketone, and toluene [84], respectively, where the authors observed an increase in the extend of degradation with a decrease in initial dyes concentration. 
Treating activated sludge, the solid content (TS) concentration may influence the $\mathrm{HC}$ effectiveness, and thus the sludge disintegration degree. Kim et al. [16] investigated the influence of initial TS concentration, ranged from 5 to $40 \mathrm{~g} \mathrm{~L}^{-1}$, on sludge solubilisation by using a Venturi system as cavitating device. The authors observed an increase in sludge solubilisation by increasing the initial TS concentration. These results are in agreement with the study carried out by Mancuso et al. [9], where the authors, ranging the initial sludge concentration from 7 to $40 \mathrm{~g} \mathrm{~L}^{-1}$, observed the highest COD solubilisation for the highest initial sludge concentration. The increase in TS content provides more cells and aggregates and thus a higher viscosity of the sludge, due to the inter- and intra- particle interactions. It was observed that both growth and collapse of cavities can be reduced by considering higher viscosities [49]. However, the increase in TS enhances the possibility of collision between sludge flocs and cavitation cavities, resulting in an increase in sludge disintegration. Therefore, the negative effect of high TS concentration on sludge degradation can be negligible if compared to the positive ones. In addition, it was observed that the presence of solids can influence the initial size of the HC nuclei [13].

\section{Influence of liquid viscosity}

The rheology of the treated matter can be characterized by its viscosity. This important parameter can influence HC intensity and the way in which a polluted liquid flows into $\mathrm{HC}$ devices. The main parts affected by viscosity are the nuclei distribution and the bubble dynamics. Generally, an increase in viscosity, regardless of whether fluids are Newtonian or nonNewtonian, can reduce the efficiency of cavitation due to a decrease in both growth and collapse of bubbles [49]. For these reasons, in most of the studied $\mathrm{HC}$ applications, water was selected as the liquid medium due to its low viscosity, which ensures higher possibilities to cavitation to occur.

However, $\mathrm{HC}$ process has been used in fluids other than water. For instance, $\mathrm{HC}$ was used as a pre-treatment technique to treat activated sludge in WWTPs. In the activated sludge, the viscosity increases with an increase in TS concentration, due to the inter- and intra-particle interactions [16]. Experimental results showed that the negative effect of viscosity on the sludge solubilisation is negligible compared to the positive effect of sludge concentration for cavitation development $[9,16]$.

When the activated sludge is treated by $\mathrm{HC}$, its viscosity decreases. Consequently, it can be heated, mixed and pumped more easily, resulting in a decrease of the supplied energy in the anaerobic digestion process [102]. A decrease in viscosity was observed by Kim et al. [16] in the first 30 min of treatment by using a Venturi system as cavitating device, whereas the viscosity was constant for a treatment time between 30 and $60 \mathrm{~min}$
Garuti et al. [97] applied HC as full-scale application to treat agro-industrial sludge, achieving a decrease in the apparent viscosity in the range of $23-27 \%$. This result was mainly attributed to an increase in temperature, shear forces and cavitation bubble collapse due to the $\mathrm{HC}$ treatment. In agreement with this study, Mancuso et al. [9] observed a decrease in viscosity by using a swirling jet-induced cavitation to increase the sludge solubilisation. As the viscosity decreased during their $\mathrm{HC}$ tests, it was necessary to progressively reduce the frequency of the pump inverter in order to keep constant the inlet pressure, and thus the flow rate and flow velocity. Using the same experimental $\mathrm{HC}$ device for treating cattle manure, Langone et al. [103] proved that the apparent viscosity dropped about 21,38 , and $40 \%$ for samples $\mathrm{HC}$ treated at 6.0, 7.0, and 8.0 bar, respectively, as compared the untreated manure. This was due to a progressive alteration of the rheology of the activated sludge, resulting in a decrease of its viscosity during the HC treatment. Similarly, Prajapat and Gogate [87] investigated the effect of inlet pressure on depolymerisation of aqueous polyacrylamide solutions by using a Venturi system. Their experimental results showed an increase in intrinsic viscosity reduction with an increase in inlet pressure. Mohod et al. [104] used an high speed homogenizer for the intensification of biodiesel production. In their study, HC combined with an increase in temperature till an optimum resulted in reduced viscosity of the oil phase and enhanced miscibility of the reactants, leading to an increase in the biodiesel yield.

\section{Influence of surface tension}

Surface tension can affect the HC process, influencing the number and the size of both vapour nuclei and gases into the liquid [74]. Higher values in surface tension lead to a lower number of gas nuclei and to a faster dissolution of them into the liquid. For this reason, at the very beginning of the HC process, cavitation efficiencies may be affected due to a low presence of gas nuclei into the liquid. However, during the collapse phase, cavities becomes nuclei and they can be cyclically used, resulting in an increase in cavitation effectiveness over time.

The surface tension depends on the type of liquid and its temperature; an increase in temperature implies a decrease in the surface tension, resulting in easier evaporation of the liquid [73]. By using very small amounts of surfactants, it is possible to reduce the surface tension and thus to decrease the cavitation threshold, resulting in an easier generation of bubbles and hydroxyl radicals.

However, while a decrease in the surface tension of the liquid can imply an easier generation of cavitation, it should also be noted that a decrease of surface tension can affect the collapse of cavities, which would be less violent. 
Influence of dissolved gas content

Cavitation nuclei are provided by the gas released due to the reduction in pressure or by the partial vaporization of liquid. The dissolved gas content can influence the rate of nucleation, and thus the cavitation intensity. Depending on the typology of the dissolved gas, different parameters such as thermal conductivity and liquid surface tension can be affected, leading to different ranges of temperature for the HC hot spots. In addition to the gas typology, also the amount of dissolved gas inside the cavity can affect the final collapse temperature and pressure [74]. The dissolved gas such as oxygen can also participate to chemical reactions that can enhance the production of free radicals. Most of the $\mathrm{HC}$ applications are carried out in the presence of gas atmosphere; however, the effect of presence of various gases (i.e. argon, air) has also been investigated [105]. It was observed that different properties of dissolved gases, such as the gas solubility, can significantly affect the cavitational process, leading to an alteration of the concentration gradient around the cavitating bubbles, and the mass transport to and from the cavitating bubbles [74].

\section{Mechanisms of degradation of pollutants}

$\mathrm{HC}$ can have both mechanical and chemical effects in the system, Fig. 12. The violent collapse of cavities in HC systems can cause the formation of hydrogen atoms and reactive hydroxyl radicals and can give rise to thermal hot spots, which can produce pyrolytic cleavage of chemicals [106]. The two main chemical mechanisms for pollutants degradation using $\mathrm{HC}$ are the thermal decomposition of volatile pollutant molecules trapped inside the cavity during the cavity collapse and, secondly, the reaction of the radicals ${ }^{\circ} \mathrm{H}$ and ${ }^{\circ} \mathrm{OH}$ with the pollutant that occurs in the cavity-water interface. In the case of non-volatile pollutants, the main mechanism for their degradation will be the attack of pollutants molecules by hydroxyl radicals in the cavity-water interface and in the bulk fluid medium.
Also, the mechanical effects are significant: (i) generation of shock waves from the collapsing cavity, (ii) creation of liquid micro-jets and (iii) formation of interfacial turbulence and powerful hydraulic tensions, due to the high flow velocity. The mechanical effects can directly break the molecular bonds on the main macromolecular chain, in particular the complex compounds of great molecular weight, thus degrading the refractory organic materials. The decomposed intermediates are more susceptible to ${ }^{\circ} \mathrm{H}$ and $\mathrm{OH}^{\circ}$ attacks and biological oxidation, which can further improve the rate of oxidation/ mineralization of pollutants [46]. Furthermore, the high velocity of micro-jets (>100 m/s [36]) disturbs the boundary layer on the solid surface, leading to the breakage of the liquid film responsible for the resistance to mass transfer.

The chemical and mechanical effects are also responsible for the improvement of heat transfer, whose rate is almost twice as high in the presence of cavitation [107].

The HC technique has been successfully applied in the field of wastewater treatment, treating contaminated wastewater and sludge and biomasses.

When $\mathrm{HC}$ is applied to contaminated wastewater the main aim is the removal of contaminants, such as dyes, pharmaceutical and toxic compounds. In this applications, chemical mechanisms, both thermal decomposition of volatile pollutant molecules and the reaction of the radicals ${ }^{\circ} \mathrm{H}$ and ${ }^{\circ} \mathrm{OH}$ with the pollutant, are prevalent as compared with the mechanical effects. However, the generated mechanical effects, as described before, can enhance the overall efficiency of the HC treatment.

$\mathrm{HC}$ is applied to activated sludge [9,21], manure and biomass $[97,103]$ as a pre-treatment, with the main purpose to improve hydrolysis and the solubilization of organic matter, and, thus, the aerobic or anaerobic biodegradability. When applied as sludge pre-treatment, $\mathrm{HC}$ acts by destroying the walls and membranes of bacterial cells with consequent release of intracellular and extracellular matter [90, 108]. Many of the intracellular constituents, including cytoplasm and nucleic acids, are readily biodegradable, resulting in acceleration of both aerobic and anaerobic digestion processes in sludge treatment or in promoting denitrification in wastewater treatment processes [9]. Furthermore, an enhancement in

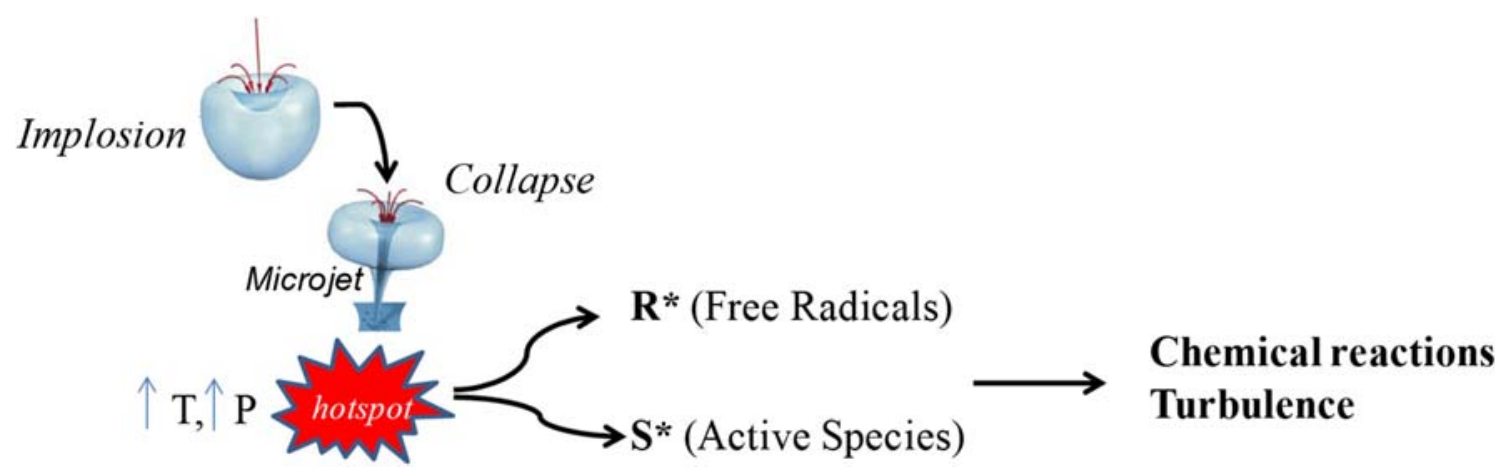

Fig. 12 Mechanism of degradation of organic pollutants 


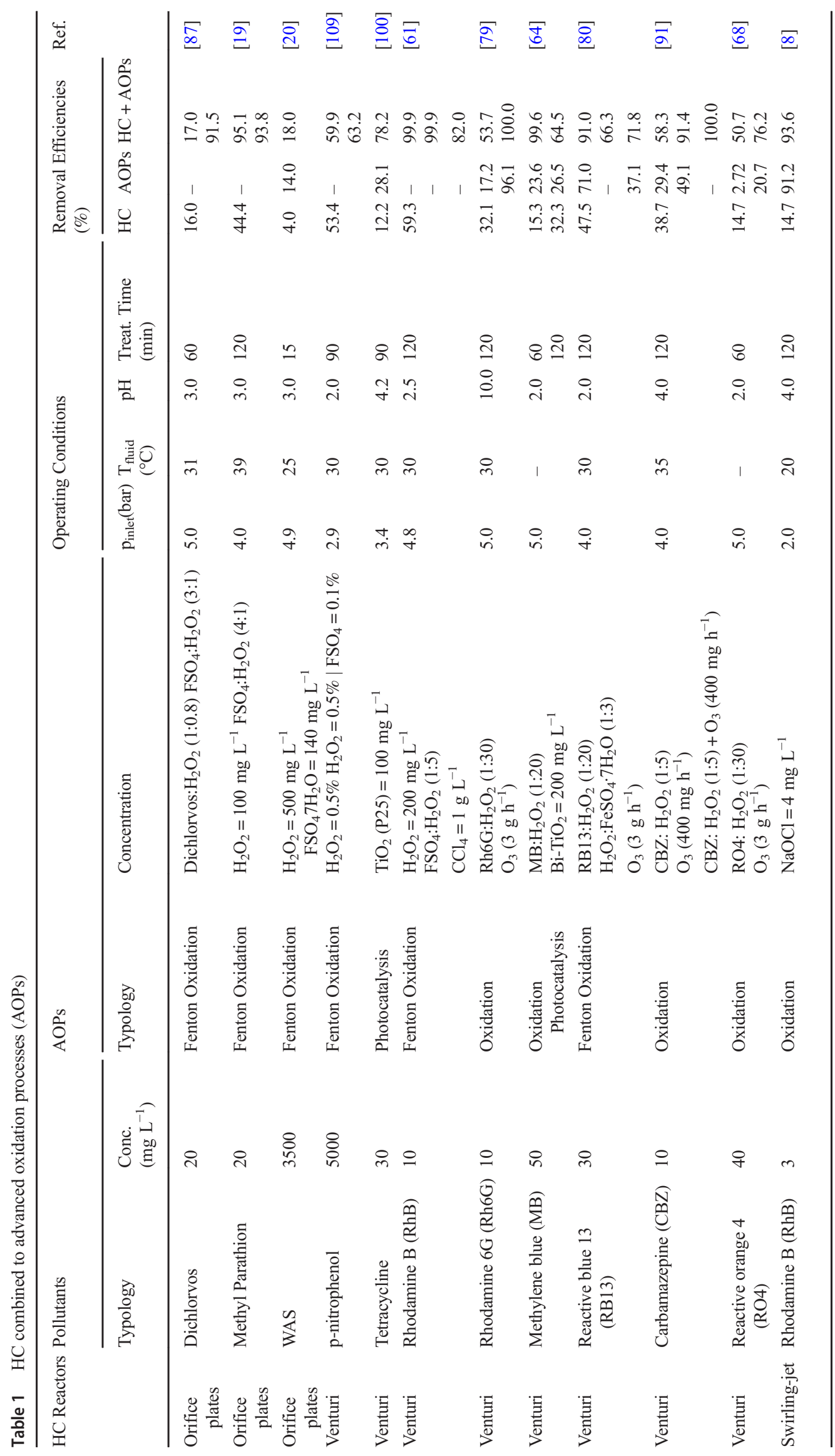


sludge dewatering performance was also observed after $\mathrm{HC}$ treatment as a result of the destruction of extra-cellular polymeric substances (EPS) and cells by highly reactive radicals [21]. When applied as biomass/manure pre-treatment, HC reduces the structural and compositional barriers present in the lignocellulosic biomass and exposes the polymer chains of cellulose and hemicellulose to microbial breakdown as well as enhances the rate of biomass degradation and biogas yield [20].

\section{HC combined to advanced oxidation processes (AOPs)}

As discussed above, $\mathrm{HC}$ has a potential in degrading organic pollutants effectively to a certain level. The efficiency of the process depends on the typology of pollutants to be treated, operating conditions, number of hydroxyl radicals that are being generated and their effective utilization.

Hence, in this regard, various researchers have investigated on the synergetic effect of HC coupled with AOPs such as $\mathrm{H}_{2} \mathrm{O}_{2}, \mathrm{O}_{3}$, Fenton's reagents, photocatalysis, etc. in order to improve the efficiency in terms of increase in 'OH radicals' generation and their effective distribution. These combined methods have shown higher efficiencies when compared to $\mathrm{HC}$ or other AOPs techniques used as individual operation.

Table 1 depicts some of the applications of $\mathrm{HC}$ combined with existing AOPs for the wastewater treatment illustrating the typology of pollutants, used equipment, operating parameters and removal efficiencies.

Further, some researcher have also investigated on the synergetic effect of $\mathrm{HC}$ in presence of additives in combination with other techniques such as acoustic cavitation $[109,110]$ and ultraviolet irradiation [92].

\section{Concluding comments}

$\mathrm{HC}$ technology seems to be very effective for intensification of chemical and mechanical processing in the specific area of wastewater treatment. Among mechanical treatments, this innovative process is taking a more prominent role, mainly due to the ease of operation, flexibility and capability to vary the required intensities of cavitational conditions.

In the present work, the importance of the $\mathrm{HC}$ phenomena in wastewater treatments engineering has been exemplified, explaining the principles of the process and critically examining the aspects related to the use of different types of HC. The efficiency of the $\mathrm{HC}$ process has been reported as function of several parameters that characterize $\mathrm{HC}$ devices and the wastewater to be treated. The optimum selection of the $\mathrm{HC}$ device and of the operating parameters will help to obtain more overall advantages for specific applications. According to the reviewed literature, there is still no fully comprehensive method to evaluate the efficiency of HC. However, some main methods commonly used for this purpose have been reported.

This work provides a useful guideline for $\mathrm{HC}$ applied to wastewater treatment and acts as a starting point for the $\mathrm{HC}$ process optimization. Moreover, the provided framework could form an origin for future literature studies, focusing on several aspects, among them modeling. Mathematical models can be used as useful tools to gain a better understanding of how $\mathrm{HC}$ devices operate in cavitating conditions and, further, to optimize performances of these devices in terms of cavitational effectiveness.

Acknowledgements This study was financially supported by the Autonomous Province of Trento, Italy (Program for the development of Small Medium Enterprise, L6/99, Project n.S155/2013/693264/12.1), and Officine Parisi s.r.1.. The second author was funded by a grant from the Fondazione Caritro, Trento (Young Researcher, Grant 2015). The authors gratefully acknowledge the technical support of Officine Parisi s.r.l. (A. Parisi and F. Parisi) and D. C. W. de Puiseau (Econovation, Germany) during the experimental activity.

\section{Compliance with ethical standards}

Declaration of interests The authors declare that they have no known competing financial interests or personal relationships that could have appeared to influence the work reported in this paper.

The authors declare the following financial interests/personal relationships which may be considered as potential competing interests:

\section{References}

1. Čudina M. Detection of cavitation phenomenon in a centrifugal pump using audible sound. 2003;17:1335-47.

2. McNulty PJ, Pearsall IS. Cavitation inception in pumps. J Fluids Eng. 1982;104:99-104.

3. Neil GD, Reuben RL, Sandford PM, Brown ER, Steel JA. Detection of incipient cavitation in pumps using acoustic emission. J Process Mech Eng. 1997;211:267-77.

4. Stepanoff AJ. Cavitation in centrifugal pumps with liquids other than water. J Eng Power. 1961;83:79-89.

5. Escaler X, Egusquiza E, Farhat M, Avellan F, Coussirat M. Detection of cavitation in hydraulic turbines. Mech Syst Signal Process. 2006;20:983-1007.

6. Ogawa K, Kimura T. Hydrodynamic characteristics of a butterfly valve-prediction of pressure loss characteristics. ISA Trans. 1995;34:319-26.

7. Batten WMJ, Bahaj AS, Molland AF, Chaplin JR. The prediction of the hydrodynamic performance of marine current turbines. Renew Energy. 2008;33:1085-96.

8. Mancuso G, Langone M, Laezza M, Andreottola G. Decolourization of Rhodamine B: a swirling jet-induced cavitation combined with $\mathrm{NaOCl}$. Ultrason Sonochem. 2016;32:18-30.

9. Mancuso G, Langone M, Andreottola G. A swirling jet-induced cavitation to increase activated sludge solubilisation and aerobic sludge biodegradability. Ultrason Sonochem. 2017;35:489-501.

10. Mancuso G, Langone M, Andreottola G, Bruni L. Effects of hydrodynamic cavitation, low-level thermal and low-level alkaline pre-treatments on sludge solubilisation. Ultrason Sonochem. 2019;59:104750. 
11. Le NT, Julcour-lebigue C, Delmas H. An executive review of sludge pretreatment by sonication. JES. 2015;37:139-53.

12. Gogate PR. A.M. Kabadi. A review of applications of cavitation in biochemical engineering / biotechnology. 2009;44:60-72.

13. Gogate PR. Cavitation: an auxiliary technique in wastewater treatment schemes. Adv Environ Res. 2002;6:335-58.

14. Sivakumar M, Pandit AB. Wastewater treatment: a novel energy efficient hydrodynamic cavitational technique. Ultrason Sonochem. 2002;9:123-31.

15. Suenaga T, Nishimura M, Yoshino H, Kato H, Nonokuchi M, Fujii $\mathrm{T}$, et al. High-pressure jet device for activated sludge reduction: feasibility of sludge solubilization. Biochem Eng J. 2015;100:1-8.

16. Kim HJ, Nguyen DX, Bae JH. The performance of the sludge pretreatment system with venturi tubes. Water Sci Technol. 2008;57:131-7.

17. Hirooka K, Asano R, Yokoyama A, Okazaki M, Sakamoto A, Nakai Y. Reduction in excess sludge production in a dairy wastewater treatment plant via nozzle-cavitation treatment: case study of an on-farm wastewater treatment plant. Bioresour Technol. 2009;100:3161-6.

18. Lee I, Han J. The effects of waste-activated sludge pretreatment using hydrodynamic cavitation for methane production. Ultrason Sonochem. 2013;20:1450-5.

19. Petkovšek M, Mlakar M, Levstek M, Strazar M, Širok B, Dular M. A novel rotation generator of hydrodynamic cavitation for wasteactivated sludge disintegration. 2015;26:408-14.

20. Patil PN, Gogate PR, Csoka L, Dregelyi-kiss A, Horvath M. Intensification of biogas production using pretreatment based on hydrodynamic cavitation. Ultrason Sonochem. 2016;30:79-86.

21. Cai M, Hu J, Lian G, Xiao R, Song Z, Jin M, et al. Synergetic pretreatment of waste activated sludge by hydrodynamic cavitation combined with Fenton reaction for enhanced dewatering. Ultrason Sonochem. 2018;42:609-18.

22. Dular M, Griessler-Bulc T, Gutierrez-Aguirre I, Heath E, Kosjek T, Krivograd Klemenčič A, et al. Use of hydrodynamic cavitation in (waste) water treatment. Ultrason Sonochem. 2016;29:577-88.

23. Didenko YT, McNamara WB, Suslick KS. Hot spot conditions during cavitation in water. J Am Chem Soc. 1999;121:5817-8.

24. Pinjari DV, Pandit AB. Cavitation milling of natural cellulose to nanofibrils. Ultrason Sonochem. 2010;17:845-52.

25. Y.T.. Shah, A.B.. Pandit, V.S. Moholkar, Cavitation reaction engineering, Luss Dan, 1999.

26. Mahulkar AV, Bapat PS, Pandit AB, Lewis FM. Steam bubble cavitation. AICHE J. 2008;54:1711-24.

27. B.T.J. Mason, J.P. Lorimer, Applied sonochemistry: the uses of power ultrasound in chemistry and processing, 2004.

28. T.J. Mason, J.P. Lorimer, Sonochemistry, theory, applications and uses of ultrasound in chemistry, in: E.H. Publishers (Ed.), Chichester, 1989: pp. 1150-1151.

29. Brennen CE. Cavitation and bubble dynamics. Oxford: Oxford University; 1995.

30. Knapp RT, Daily JW, Hammit FG. Cavitation. New York: McGraw-Hill; 1970.

31. Pilli S, Bhunia P, Yan S, Leblanc RJ, Tyagi RD, Surampalli RY. Ultrasonic pretreatment of sludge: a review. Ultrason Sonochem. 2011;18:1-18.

32. Gogate PR, Pandit AB. Engineering design methods for cavitation reactors II: hydrodynamic cavitation. AICHE J. 2000;46:1641-9.

33. Delmas H, Le NT, Barthe L, Julcour-lebigue C. Optimization of hydrostatic pressure at varied sonication conditions - power density, intensity, very low frequency - for isothermal ultrasonic sludge treatment. Ultrason Sonochem. 2015;25:51-9.

34. F. Avellan, M. Farhat, Shock pressure generated by cavitation vortex collapse, in: Proc. Third Onternational Symp. Cavitation Noise Eros. Fluid Syst., FED-vol 88, ASME Winter Annual Meeting San Francisco, CA, 1989: pp. 119-125.
35. Akhatov I, Lindau O, Topolnikov A, Mettin R, Vakhitova N, Lauterborn W. Collapse and rebound of a laser-induced cavitation bubble. Phys Fluids. 2001;13:2805-19.

36. J.-P. Franc, J.-M. Michel, Foundamentals of cavitation, 2005.

37. Riesz P, Berdahl D, Christman CL. Free radical generation by ultrasound in aqueous and nonaqueous solutions. Environ Health Perspect. 1985;64:233-52.

38. Adewuyi YG. Critical Review sonochemistry in environmental remediation. 1. Combinative and hybrid sonophotochemical oxidation processes for the treatment of pollutants in water. Environ. Sci. Technol. 2005;39:3409-20.

39. Farhat M, Chakravarty A, Field E. Luminescence from hydrodynamic cavitation. Math Phys Eng Sci. 2011;467:591-606.

40. Sunartio D, Ashokkumar M, Grieser F. Study of the coalescence of acoustic bubbles as a function of frequency, power, and watersoluble additives. J Am Chem Soc. 2007;129:6031-6.

41. Zhao H, Wang J-X, Wang Q-A, Chen J-F, Yun J. Controlled liquid antisolvent precipitation of hydrophobic pharmaceutical nanoparticles in a microchannel reactor. Ind Eng Chem Res. 2007;46: 8229-35.

42. Jiju JM. Application of advanced oxidation processes for the degradation of organic water pollutants. Kottayam: Mahtama Gandhi University; 2000.

43. Moholkar VS, Pandit AB. Bubble behavior in hydrodynamic cavitation: Effect of turbulence. AIChE J. 1997;43:1641-8.

44. Yan Y, Thorpe RB. Flow regime transitions due to cavitation in the flow through an orifice. Int J Multiph Flow. 1990;16:1023-45.

45. K.K. Jyoti, A.B. Pandit, Water disinfection by acoustic and hydrodynamic cavitation, 7 (2001) 201-212.

46. Saharan VK, Badve MP, Pandit AB. Degradation of reactive red 120 dye using hydrodynamic cavitation. Chem Eng J. 2011;178: $100-7$.

47. Šarc A, Stepišnik-Perdih T, Petkovšek M, Dular M. The issue of cavitation number value in studies of water treatment by hydrodynamic cavitation. 2017;34:51-9.

48. Petkovšek M, Zupanc M, Dular M, Kosjek T, Heath E, Kompare $\mathrm{B}$, et al. Rotation generator of hydrodynamic cavitation for water treatment. Sep Purif Technol. 2013;118:415-23.

49. F. Jean-Pierre, M. Jean-Marie, Fundamentals of cavitation, 2004.

50. Choi J, Hsiao C, Chahine G, Ceccio S. Growth, oscillation and collapse of vortex cavitation bubbles. J Fluid Mech. 2009;624: 255-79.

51. Palau-Salvador G, González-Altozano P, Arviza-Valverde J. Numerical modeling of cavitating flows for simple geometries using FLUENT V6. 1, Spanish. J Agric Res. 2007;5:460-9.

52. Simpson A, Ranade VV. Modelling of hydrodynamic cavitation with orifice: influence of different orifice designs. Chem Eng Res Des. 2018;136:698-711.

53. Pawar SK, Mahulkar AV, Pandit AB, Roy K, Moholkar VS. Sonochemical effect induced by hydrodynamic cavitation: comparison of Venturi/orifice flow geometries. VTT Publ. 2017;63: 4705-16.

54. Navickas J, Chen L. Cavitating venturi performance characteristics. ASME Fluids Eng Div. 1993;177:153-9.

55. Wang Q, Jiang Z. Effect of nozzle geometrical and dynamic factors on cavitating and turbulent flow in a diesel multi-hole injector nozzle. Int J Therm Sci. 2013;70:132-43.

56. S.B. Müller, L. Kleiser, Large-Eddy simulation of vortex breakdown in compressible swirling jet flow, in: Conf. Turbolence Interact., 2006.

57. Ashrafizadeh SM, Ghassemi H. Experimental and numerical investigation on the performance of small-sized cavitating venturis. Flow Meas Instrum. 2015;42:6-15.

58. Badve MP, Alpar T, Pandit AB, Gogate PR, Csoka L. Modeling the shear rate and pressure drop in a hydrodynamic cavitation 
reactor with experimental validation based on KI decomposition studies. 2015;22:272-7.

59. Mancuso G. Experimental and numerical investigation on performance of a swirling jet reactor. Ultrason Sonochem. 2018;49:2418.

60. Wang X, Wang J, Guo P, Guo W, Li G. Chemical effect of swirling jet-induced cavitation: degradation of rhodamine $\mathrm{B}$ in aqueous solution. Ultrason Sonochem. 2008;15:357-63.

61. Wang X, Wang J, Guo P, Guo W, Wang C. Degradation of rhodamine $\mathrm{B}$ in aqueous solution by using swirling jet-induced cavitation combined with H2O2. J Hazard Mater. 2009;169:486-91.

62. Mishra KP, Gogate PR. Intensification of degradation of Rhodamine B using hydrodynamic cavitation in the presence of additives. Sep Purif Technol. 2010;75:385-91.

63. Gogate PR, Bhosale GS. Comparison of effectiveness of acoustic and hydrodynamic cavitation in combined treatment schemes for degradation of dye wastewaters. Chem Eng Process Process Intensif. 2013;71:59-69.

64. Wang J, Guo Y, Guo P, Yu J, Guo W, Wang X. Degradation of reactive brilliant red $\mathrm{K}-2 \mathrm{BP}$ in water using a combination of swirling jet-induced cavitation and Fenton process. Sep Purif Technol. 2014;130:1-6.

65. Kumar MS, Sonawane SH, Pandit AB. Degradation of methylene blue dye in aqueous solution using hydrodynamic cavitation based hybrid advanced oxidation processes. Chem Eng Process Process Intensif. 2017;122:288-95.

66. Zupanc M, Kosjek T, Petkovšek M, Dular M, Kompare B, Širok $\mathrm{B}$, et al. Removal of pharmaceuticals from wastewater by biological processes, hydrodynamic cavitation and UV treatment. Ultrason Sonochem. 2013;20:1104-12.

67. Zupanc M, Kosjek T, Petkovšek M, Dular M, Kompare B, Sirok $\mathrm{B}$, et al. Shear-induced hydrodynamic cavitation as a tool for pharmaceutical micropollutants removal from urban wastewater. Ultrason. Sonochem. 2013:18-9.

68. Gogate PR, Shirgaonkar IZ, Sivakumar M, Senthilkumar P, Vichare NP, Pandit AB. Cavitation reactors: efficiency assessment using a model reaction. AICHE J. 2001;47:2526-38.

69. Gore MM, Saharan VK, Pinjari DV, Chavan PV, Pandit AB. Degradation of reactive orange 4 dye using hydrodynamic cavitation based hybrid techniques. Ultrason Sonochem. 2014;21:107582.

70. Habashi N, Mehrdadi N, Mennerich A, Alighardashi A, Torabian A. Hydrodynamic cavitation as a novel approach for pretreatment of oily wastewater for anaerobic co-digestion with waste activated sludge. Ultrason Sonochem. 2016;31:362-70.

71. Zhang G, Zhang P, Yang J, Liu H. Energy-efficient sludge sonication: power and sludge characteristics. Bioresour Technol. 2008;99:9029-31.

72. Braeutigam P, Franke M, Wu ZL, Ondruschka B. Role of different parameters in the optimization of hydrodynamic cavitation. Chem Eng Technol. 2010;33:932-40.

73. J. Ozonek, Application of hydrodynamic cavitation in environmental engineering, 2012.

74. Gogate PR, Pandit AB. Hydrodynamic cavitation reactors: a state of the art review. Chem Eng. 2001;17:1-85.

75. S. Manickam, M. Ashokkumar, Cavitation: A Novel EnergyEfficient Technique for the Generation of Nanomaterials, 2014.

76. Chakinala AG, Gogate PR, Burgess AE, Bremner DH. Treatment of industrial wastewater effluents using hydrodynamic cavitation and the advanced Fenton process. 2008;15:49-54.

77. Wang J, Wang X, Guo P, Yu J. Degradation of reactive brilliant red $\mathrm{K}-2 \mathrm{BP}$ in aqueous solution using swirling jet-induced cavitation combined with H2O2. Ultrason Sonochem. 2011;18:494-500.

78. Basiri Parsa J, Ebrahimzadeh Zonouzian SA. Optimization of a heterogeneous catalytic hydrodynamic cavitation reactor performance in decolorization of Rhodamine B: application of scrap iron sheets. Ultrason Sonochem. 2013;20:1442-9.

79. Ozonek J, Lenik K. Effect of different design features of the reactor on hydrodynamic cavitation process. Arch Mater Sci Eng. 2011;52:112-7.

80. Rajoriya S, Bargole S, Saharan VK. Degradation of a cationic dye (Rhodamine 6G) using hydrodynamic cavitation coupled with other oxidative agents: reaction mechanism and pathway. Ultrason Sonochem. 2017;34:183-94.

81. Rajoriya S, Bargole S, Saharan VK. Degradation of reactive blue 13 using hydrodynamic cavitation: effect of geometrical parameters and different oxidizing additives. Ultrason. - Sonochemistry. 2017;37:192-202.

82. Carpenter J, George S, Saharan VK. Low pressure hydrodynamic cavitating device for producing highly stable oil in water emulsion: effect of geometry and cavitation number. Chem Eng Process Process Intensif. 2017;116:97-104.

83. Langone M, Ferrentino R, Trombino G, Puiseau WDE, Andreottola G, Rada EC, et al. Application of a Novel Hydrodynamic Cavitation System in Wastewater Treatment Plants. 2013;77:225-34.

84. Suryawanshi PG, Bhandari VM, Sorokhaibam LG, Ruparelia JP, Ranade VV. Solvent degradation studies using hydrodynamic cavitation. Environ Prog Sustain Energy. 2017;37:295-304.

85. Badve M, Gogate P, Pandit A, Csoka L. Hydrodynamic cavitation as a novel approach for wastewater treatment in wood finishing industry. Sep Purif Technol. 2013;106:15-21.

86. Jain P, Bhandari VM, Balapure K, Jena J, Ranade VV, Killedar DJ. Hydrodynamic cavitation using vortex diode: an efficient approach for elimination of pathogenic bacteria from water. $\mathrm{J}$ Environ Manag. 2019;242:210-9.

87. Prajapat AL, Gogate PR. Intensified depolymerization of aqueous polyacrylamide solution using combined processes based on hydrodynamic cavitation, ozone, ultraviolet light and hydrogen peroxide. Ultrason Sonochem. 2016;31:371-82.

88. Joshi RK, Gogate PR. Degradation of dichlorvos using hydrodynamic cavitation based treatment strategies. Ultrason Sonochem. 2012;19:532-9.

89. Wang X, Zhang Y. Degradation of alachlor in aqueous solution by using hydrodynamic cavitation. J Hazard Mater. 2009;161:202-7.

90. Xie L, Terada A, Hosomi M. Disentangling the multiple effects of a novel high pressure jet device upon bacterial cell disruption. Chem Eng J. 2017;323:105-13.

91. Kumar PS, Pandit AB. Modeling hydrodynamic cavitation. Chem Eng Technol. 1999;22:1017-27.

92. Thanekar P, Panda M, Gogate PR. Degradation of carbamazepine using hydrodynamic combined with advanced oxidation processes. Ultrason - Sonochemistry. 2018;40:567-76.

93. Choi J, Cui M, Lee Y, Kim J, Son Y, Khim J. Hydrodynamic cavitation and activated persulfate oxidation for degradation of bisphenol a: kinetics and mechanism. Chem Eng J. 2018;338: 323-32.

94. Tao Y, Cai J, Huai X, Liu B. A novel device for hazardous substances degradation based on double-cavitating-jets impingement: parameters optimization and efficiency assessment. J Hazard Mater. 2017;335:188-96.

95. Merouani S, Hamdaoui O, Saoudi F, Chiha M. Sonochemical degradation of Rhodamine $\mathrm{B}$ in aqueous phase: effects of additives. Chem Eng J. 2010;158:550-7.

96. Grübel K, Suschka J. Hybrid alkali-hydrodynamic disintegration of waste-activated sludge before two-stage anaerobic digestion process. Environ Sci Pollut Res. 2015;22:7258-70.

97. Garuti M, Langone M, Fabbri C, Piccinini S. Monitoring of fullscale hydrodynamic cavitation pretreatment in agricultural biogas plant. Bioresour Technol. 2018;247:599-609. 
98. Floury J, Legrand J, Desrumaux A. Analysis of a new type of high pressure homogeniser. Part B. study of droplet break-up and recoalescence phenomena. Chem Eng Sci. 2004;59:1285-94.

99. Tang SY, Sivakumar M. A novel and facile liquid whistle hydrodynamic cavitation reactor to produce submicron multiple emulsions. Am Inst Chem Eng. 2012:1-13.

100. Save SS, Pandit AB, Joshi JB. Use of hydrodynamic cavitation for large scale microbial cell disruption. IChemE. 1997;75:41-9.

101. Wang X, Jia J, Wang Y. Combination of photocatalysis with hydrodynamic cavitation for degradation of tetracycline. Chem Eng J. 2017;315:274-82.

102. Montgomery LFR, Bochmann G. Pretreatment of feedstock for enhanced biogas production. IEA Bioenergy. 2014:1-20.

103. Langone M, Soldano M, Fabbri C, Pirozzi F, Andreottola G. Anaerobic digestion of cattle manure influenced by swirling jet induced hydrodynamic cavitation. Appl Biochem Biotechnol. 2017;184:1200-18.

104. Mohod AV, Gogate PR, Viel G, Firmino P, Giudici R. Intensification of biodiesel production using hydrodynamic cavitation based on high speed homogenizer. Chem Eng J. 2017;316: 751-7.

105. Kumar KS, Moholkar VS. Conceptual design of a novel hydrodynamic cavitation reactor. Chem Eng Sci. 2007;62:2698-711.
106. Gogate PR, Pandit AB. A review of imperative technologies for wastewater treatment I: oxidation technologies at ambient conditions. Adv Environ Res. 2004;8:501-51.

107. Kimoto H, Sumita Y. Heat transfer characteristics of a circular cylinder in a conduit under cavitation. Trans JSME. 1986;49: 2312-3.

108. Feng X, Lei H, Deng J, Yu Q, Li H. Chemical Engineering and Processing : Process Intensification Physical and chemical characteristics of waste activated sludge treated ultrasonically. 2009;48: 187-94.

109. Yi C, Lu Q, Wang Y, Wang Y, Yang B. Degradation of organic wastewater by hydrodynamic cavitation combined with acoustic cavitation. Ultrason Sonochem. 2018;43:156-65.

110. Cai MQ, Hu JQ, Wells G, Seo Y, Spinney R, Ho SH, et al. Understanding mechanisms of synergy between acidification and ultrasound treatments for activated sludge dewatering: from bench to pilot-scale investigation. Environ Sci Technol. 2018;52: 4313-23.

Publisher's note Springer Nature remains neutral with regard to jurisdictional claims in published maps and institutional affiliations. 